\title{
Witt Groups of Algebraic Groups
}

\author{
by
}

\author{
Nobuaki YAGITA
}

\begin{abstract}
Let $G_{k}$ be a split reductive group over a subfield $k$ of $\mathbb{C}$ corresponding to a Lie group $G$. Let $T$ be a maximal torus of $G$. We show the isomorphism $W^{*}\left(G_{k}\right) \cong W^{*}\left(G_{k} / T_{k}\right)$ of Balmer-Witt groups. When $k$ is algebraically closed, we prove that $W^{*}\left(G_{k}\right)$ is isomorphic to the topological $K$-theory $K O^{2 *-1}(G / T)$ of the flag manifold $G / T$. Then we compute it explicitly by using the fact that $W^{*}\left(G_{k}\right)$ is a Hopf algebra.
\end{abstract}

2010 Mathematics Subject Classification: Primary 19G12, 55N15; Secondary 55N20.

Keywords: algebraic group, Witt group, $K$-theory.

\section{$\S 1$. Introduction}

Let $X$ be a (quasi projective) smooth variety over a field $k \subset \mathbb{C}$. The Witt group $W(X)$ is the quotient of the Grothendieck group of vector bundles with quadratic forms over $X$, by the subgroup generated by bundles with quadratic forms which admit Lagrangian subbundles. The generalized Witt group $W^{*}(X ; L)$ was defined by Balmer [Ba] for $* \in \mathbb{Z} / 4$ and for a line bundle $L$ on $X$ so that $W^{0}\left(X ; O_{X}\right)=W(X)$. Let us write simply $W^{*}(X)$ for $W^{*}\left(X, O_{X}\right)$. We can define a natural map ([Ya3], [Zi])

$$
q^{*}: W^{*}(X) \rightarrow K O^{2 *}(X(\mathbb{C})) / K U^{2 *}(X(\mathbb{C})) \stackrel{\times \eta}{\longrightarrow} K O^{2 *-1}(X(\mathbb{C}))
$$

where $K O^{*}(-)$ and $K U^{*}(-)$ are (topological) real and complex $K$-theories, and $0 \neq \eta \in K O^{-1}(\mathrm{pt}) \cong \mathbb{Z} / 2$.

Let $G$ be a compact simply connected Lie group, $T$ a maximal torus of $G$, and $B$ the Borel subgroup with $T \subset B$. Let us denote by $G_{k}$ (resp. $T_{k} \subset B_{k}$ ) the split reductive group (resp. the split maximal torus and the Borel subgroup

Communicated by T. Ohtsuki. Received October 6, 2011. Revised October 26, 2012, March 14, 2013, July 18, 2013 and September 5, 2013.

N. Yagita: Department of Mathematics, Faculty of Education, Ibaraki University, Mito, Ibaraki, Japan;

e-mail: yagita@mx.ibaraki.ac.jp 
containing it) over $k$ which corresponds to $G$ (resp. T, B). Let

$$
T_{k}^{1} \subset T_{k}^{2} \subset \cdots \subset T_{k}^{\ell}=T_{k}
$$

be a sequence of tori of $G_{k}$ where $T_{k}^{i} \cong\left(\mathbb{A}^{1}-\{0\}\right)^{\times i}$. Recently Calmès and Fasel [Ca-Fa] proved that $W^{*}\left(G_{k} / B_{k} ; L\right)=0$ whenever $0 \neq L \in \operatorname{Pic}\left(G_{k} / T_{k}\right) / 2$. (Note that $W^{*}\left(G_{k} / B_{k} ; L\right) \cong W^{*}\left(G_{k} / T_{k} ; L\right)$ for all $L$.) There is a localization exact sequence in Witt theory

$$
\stackrel{\delta}{\rightarrow} W^{*-1}\left(G_{k} / T_{k}^{i} ; t_{i}\right) \stackrel{j_{*}}{\rightarrow} W^{*}\left(G_{k} / T_{k}^{i}\right) \rightarrow W^{*}\left(G_{k} / T_{k}^{i-1}\right) \stackrel{\delta}{\rightarrow} \cdots
$$

where $\left(t_{1}, \ldots, t_{n}\right)$ is a basis of $\operatorname{Pic}\left(G_{k} / T_{k}\right) / 2$. Here $W^{*-1}\left(G_{k} / T_{k}^{i} ; t_{i}\right)=0$ from the result by Calmès and Fasel. By induction on $i$, we obtain

Lemma 1.1 (Theorem 2.3). If $G$ is a simply connected Lie group, then $W^{*}\left(G_{k}\right) \cong$ $W^{*}\left(G_{k} / T_{k}\right)$.

Now let $k$ be an algebraically closed subfield in $\mathbb{C}$. Also recently, Zibrowius [Zi] showed that the above map $q^{*}$ is always an isomorphism for each cellular variety $X$ (which can be decomposed as $\coprod_{s} \mathbb{A}^{i_{s}}$, see $\S 5$ for the definition) when $k=\mathbb{C}$, and it can be generalized to any algebraically closed field in $\mathbb{C}$. Of course, the flag variety $G_{k} / B_{k}$ is cellular. Hence we have the isomorphism (from the Bott exact sequence (4.2), [At], [Ko-Ha])

$$
q^{*}: W^{*}\left(G_{k}\right) \cong K O^{2 *}(G / T) / K U^{2 *}(G / T) \cong K O^{2 *-1}(G / T) .
$$

Consider the Atiyah-Hirzebruch spectral sequence converging to $K O^{*}(G / T)$. It is known that the differential $d_{2}$ is the Steenrod squaring $\mathrm{Sq}^{2} \bmod 2([\mathrm{Ha}],[\mathrm{Fu}])$. Kishimoto, Kono and Ohsita [Ki-Ko-Oh], [Ki-Oh], [Oh] computed this spectral sequence and showed that it collapses from the $E_{3}$-term for compact simply connected simple Lie groups except $E_{7}$ and $E_{8}$. Moreover the above $K O^{2 *-1}(G / T)$ is isomorphic to

$$
H^{*}\left(G / T ; \mathrm{Sq}^{2}\right)=H\left(H^{2 *}(G / T ; \mathbb{Z} / 2) ; \mathrm{Sq}^{2}\right)
$$

for these Lie groups.

On the other hand, there are spectral sequences $E(\mathrm{GP})_{r}^{*, *^{\prime}}$ (by Gille and Pardon $[\mathrm{Gi}],[\mathrm{Pa}])$ and $E(\mathrm{BW})_{r}^{*, *^{\prime}}$ (by Balmer-Walter [Ba-Wa]) such that $E(\mathrm{GP})_{r}^{*, *^{\prime}}$ converges to $E(\mathrm{BW})_{2}^{*, 4 c}$ (for $c \in \mathbb{Z}$ ), and $E(\mathrm{BW})_{r}^{*, *^{\prime}}$ converges to $W^{*+*^{\prime}}\left(G_{k}\right)$.

By the Borel theorem (see $[\mathrm{Ka}]$ for example), the cohomology $H^{*}(G ; \mathbb{Z} / 2)$ is isomorphic to a product of truncated polynomial algebras generated by odd dimensional elements $x_{i}$. Let us write $y_{j}=x_{j}^{2}$ if it is not zero. We consider the graded ring gr $H^{*}(G ; \mathbb{Z} / 2)=\bigoplus_{i} F^{i+1} / F^{i}$ defined by the filtration $F^{i}$ giving degree 0 (resp. 1) to $y_{j}$ (resp. $x_{j}$ ). Then $\mathrm{Sq}^{2}$ acts on $\operatorname{gr} H^{*}(G ; \mathbb{Z} / 2)$ as a differential. Let us denote its 
homology by

$$
H^{*}\left(G ; \mathrm{Sq}^{2}\right)=H\left(\operatorname{gr} H^{*^{\prime \prime}}(G ; \mathbb{Z} / 2) ; \mathrm{Sq}^{2}\right)
$$

where the degree in $H^{*}\left(G ; \mathrm{Sq}^{2}\right)$ is given by

$$
\operatorname{deg}\left(y_{i}\right)=\frac{1}{2}\left|y_{i}\right| \quad \text { and } \quad \operatorname{deg}\left(x_{j}\right)=\frac{1}{2}\left(\left|x_{j}\right|-1\right)
$$

where $|a|=*^{\prime \prime}$ is the degree of $a \in H^{*^{\prime \prime}}(G ; \mathbb{Z} / 2)$. We will prove that $E(\mathrm{GP})_{3}^{*, *^{\prime}} \cong$ $H^{*}\left(G ; \mathrm{Sq}^{2}\right)$ so that the bidegree of $E(\mathrm{GP})_{r}^{*, *^{\prime}}$ is given by

$$
\operatorname{deg}\left(y_{i}\right)=\left(\frac{1}{2}\left|y_{i}\right|, \frac{1}{2}\left|y_{i}\right|\right) \quad \text { and } \operatorname{deg}\left(x_{j}\right)=\left(\frac{1}{2}\left(\left|x_{j}\right|-1\right), \frac{1}{2}\left(\left|x_{j}\right|+1\right)\right) .
$$

In fact, from Totaro [To], it is known that $d_{2}=\mathrm{Sq}^{2}$ on $E(\mathrm{GP})_{2}^{*, *^{\prime}}$ when $*=*^{\prime}$. Moreover we will prove

Theorem 1.2 (Theorem 6.9). Let $k$ be an algebraically closed subfield of $\mathbb{C}$. For each compact connected simple Lie group $G$, the Atiyah-Hirzebruch spectral sequence converging to $\mathrm{KO}^{*}(G / T)$ collapses from the $E_{3}$-term. If $G$ is simply connected, then we have isomorphisms

$$
W^{*}\left(G_{k}\right) \cong H^{*}\left(G / T ; \mathrm{Sq}^{2}\right) \cong \Lambda\left(z_{1}, \ldots, z_{s}\right), \quad \operatorname{deg}\left(z_{i}\right) \text { odd },
$$

where $\Lambda\left(z_{1}, \ldots, z_{s}\right)$ is the exterior algebra over $\mathbb{Z} / 2$ generated by $z_{1}, \ldots, z_{s}$. For $G=S O(n)$, we have an isomorphism

$$
W^{*}\left(G_{k}\right) \cong H^{*}\left(G / T ; \mathrm{Sq}^{2}\right) \otimes \Lambda\left(x_{0}\right) \cong \Lambda\left(x_{0}, z_{1}, \ldots, z_{r}\right), \quad \operatorname{deg}\left(x_{0}\right)=0 .
$$

Moreover $W^{*}\left(G_{k}\right)$ is also isomorphic to

$$
\begin{cases}E(\mathrm{GP})_{4}^{*, *^{\prime}} \cong H\left(H^{*}\left(G ; \mathrm{Sq}^{2}\right) ; d_{3}\right) & \text { for } G=E_{6}, E_{7}, E_{8}, \\ E(\mathrm{GP})_{3}^{*, *^{\prime}} \cong H^{*}\left(G ; \mathrm{Sq}^{2}\right) & \text { for other simple Lie groups. }\end{cases}
$$

Since $W^{*}\left(G_{k}\right)$ and $H^{*}\left(G / T ; \mathrm{Sq}^{2}\right)$ satisfy the Künneth formula (Corollary 4.3), the above theorem holds for all Lie groups $G$ which are products of compact connected simple Lie groups (e.g. compact simply connected Lie groups since $G / T$ is then a product of $G_{i} / T_{i}$ for simple Lie groups $G_{i}$ and their maximal tori $\left.T_{i}\right)$. The explicit value of $\operatorname{deg}\left(z_{i}\right)$ is given in $\S 7$ below.

This paper is organized as follows. In $\S 2$, we recall the properties of the BalmerWitt group $W^{*}\left(G_{k}\right), W^{*}\left(G_{k} / T_{k}\right)$, and we prove Lemma 1.1. In $\S 3$, we compute the motivic cohomology $H^{*, *^{\prime}}\left(G_{k} ; \mathbb{Z} / 2\right)$ from $H^{*, *^{\prime}}\left(G_{k} / T_{k} ; \mathbb{Z} / 2\right)$. In $\S 4$, we recall the (topological) real and complex $K$-theories $K O^{*}(G / T)$ and $K U^{*}(G / T)$, and study their Atiyah-Hirzebruch spectral sequences. In $\S 5$, we identify $W^{*}\left(G_{k}\right)$ with the (motivic) hermitian $K$-theory $K O^{*, *^{\prime}}\left(G_{k}\right)$, and we give topological proofs of results by Zibrowius [Zi] and Calmès-Fasel [Ca-Fa]. In $\S 6$, we study the Balmer-Walter and 
Gille-Pardon spectral sequences for $G_{k}$, and show that each page of the spectral sequences is a differential Hopf algebra. Using these, we prove the main theorem (Theorem 1.2) under the assumptions of some concrete computations for simple Lie groups which are given in $\S 7$.

\section{$\S 1.1$. Notations}

The following notations are used throughout this paper.

- $k$ : a subfield of $\mathbb{C}(\operatorname{ch}(k) \neq 2$ in $\S 2) ; X$ : a smooth $k$-variety; $X(\mathbb{C})$ : the complex manifold of $\mathbb{C}$-rational points.

- $G$ : a Lie group (a linear algebraic group in $\S 2) ; G_{k}$ : the reductive algebraic group over $k$ corresponding to $G ; T=T^{\ell}$ : a maximal torus of $G ; T_{k}$ : the split maximal torus of $G_{k}$.

- $W^{*}(X), W^{*}(X ; L)$ : the Balmer-Witt group (with coefficients $\left.L\right) ; K O^{*}(X(\mathbb{C})$ ), $K U^{*}(X(\mathbb{C}))$ : the (topological) real and complex $K$-theories; $K O^{*, *^{\prime}}(X)$ : the (motivic) hermitian $K$-theory.

- $\operatorname{Pic}(X)=\mathrm{CH}^{1}(X)$ : the Picard group; $\mathrm{CH}^{i}(X)$ : the Chow group of codimension $i$ algebraic cycles modulo rational equivalence; $H^{*, *^{\prime}}(X ; \mathbb{Z} / 2)$ : the $\bmod (2)$ motivic cohomology over $k$ defined by Suslin and Voevodsky [Vo1, Vo2] so that $H^{2 *, *}(X ; \mathbb{Z} / 2) \cong \mathrm{CH}^{*}(X) / 2 ; H_{\mathrm{Zar}}^{*}\left(X ; H_{\mathbb{Z} / 2}^{*^{\prime}}\right)$ : the sheaf cohomology where $H_{\mathbb{Z} / 2}^{*^{\prime}}$ is the sheaf defined from the presheaf $U \mapsto H_{\mathrm{et}}^{*^{\prime}}(U ; \mathbb{Z} / 2)$ for open sets $U$ in $X$.

- $H^{*}\left(A ; \mathrm{Sq}^{2}\right)$ : the homology of the $\mathrm{Sq}^{2}$-module $A$ with the differential $d=$ $\mathrm{Sq}^{2}$. In particular, we simply write $H^{*}\left(G / T ; \mathrm{Sq}^{2}\right)$ and $H^{*}\left(G ; \mathrm{Sq}^{2}\right)$ when $A=$ $H^{*}(G / T ; \mathbb{Z} / 2)$ and $A=\operatorname{gr} H^{*}(G ; \mathbb{Z} / 2)$ respectively.

- $E(\mathrm{BW})_{r}^{*, *^{\prime}}, E(\mathrm{GP})_{r}^{*, *^{\prime}}$ : the Balmer-Walter and the Gille-Pardon spectral sequences (e.g. $\left.E(\mathrm{GP})_{2}^{*, *^{\prime}} \cong H_{\text {Zar }}^{*}\left(-; H_{\mathbb{Z} / 2}^{*^{\prime}}\right)\right)$.

- $\Lambda\left(x_{1}, \ldots, x_{\ell}\right)$ : the exterior algebra generated by odd-dimensional elements $x_{i}$; $P(y)$ : the truncated polynomial algebra generated by even-dimensional elements $y_{i}$, such that gr $H^{*}(G ; \mathbb{Z} / 2) \cong P(y) \otimes \Lambda\left(x_{1}, \ldots, x_{\ell}\right) ; S(t)=H^{*}(B T ; \mathbb{Z} / 2)$ : the polynomial algebra generated by $t_{1}, \ldots, t_{\ell}$ such that $\operatorname{gr} H^{*}(G / T ; \mathbb{Z} / 2) \cong$ $P(y) \otimes S(t) /\left(b_{1}, \ldots, b_{\ell}\right)$ for some $b_{i} \in S(t)$.

- $A\left\{a_{1}, \ldots, a_{n}\right\}$ : the free $A$-module generated by $a_{1}, \ldots, a_{n}$.

\section{§2. Balmer-Witt group}

In this section, we assume that $X$ is a smooth scheme over a field $k$ of characteristic $\operatorname{ch}(k) \neq 2$ (while in other sections, we assume $\operatorname{ch}(k)=0$ ). We can consider the generalized Witt group $W^{i}(X ; L)$ for $i \in \mathbb{Z} / 4$ and for a line bundle $L$ on $X$ such that 
the usual Witt group $W^{i}(X)$ is $W^{i}\left(X ; O_{X}\right)$ (see [Ba], [Ba-Ca2], [Zi] for example) where $O_{X}$ is the line bundle corresponding to the structural sheaf over $X$. We also note that if $L=L^{\prime} \bmod (2)$, then we have a (noncanonical) isomorphism $W^{*}(X ; L) \cong W^{*}\left(X ; L^{\prime}\right)$. Let us write the total Witt group

$$
W^{\text {total }}(X)=\bigoplus_{i \in \mathbb{Z} / 4, L \in \operatorname{Pic}(X) / 2} W^{i}(X ; L) .
$$

The Gysin and the boundary maps were defined as maps of $W^{\text {total }}(-)$. (Gysin maps are constructed by Calmès and Hornbostel [Ca-Ho], see also [Ne, §4.1].) Let $g: Z \hookrightarrow X$ be a regular embedding of codimension $c$, and $f: U=X-Z \hookrightarrow X$ Let $\omega_{g}$ be the relative canonical bundle (for the definition see [Ba-Ca1]). Then we have the natural exact sequence [Ba-Ca1, (11)]

$$
\rightarrow W^{*-c}\left(Z ;\left.\omega_{g} \otimes L\right|_{Z}\right) \stackrel{g_{*}}{\longrightarrow} W^{*}(X ; L) \stackrel{f^{*}}{\rightarrow} W^{*}\left(U ;\left.L\right|_{U}\right) \stackrel{\delta}{\rightarrow} \cdots .
$$

Let $G_{k}$ be a split reductive group over a field $k$ corresponding to an algebraic group $G$ (for definitions and properties of algebraic groups, see [Bo] for example.) Let $T_{k}$ be a maximal (split) torus of $G_{k}$. We consider the sequence of tori

$$
T_{k}^{1} \subset T_{k}^{2} \subset \cdots \subset T_{k}^{\ell}=T_{k} \text { where } T_{k}^{i} \cong\left(\mathbb{A}^{1}-\{0\}\right)^{\times i} \cong\left(\mathbb{G}_{\mathrm{m}}\right)^{\times i} .
$$

Let $Z$ be the central torus of $G_{k}$. Let $G_{\mathrm{ss}}=G_{k} / Z$ be the semisimple quotient of $G_{k}$ with the maximal (split) torus $T_{\mathrm{ss}}=T_{k} / Z$, let $G_{\mathrm{sc}}$ be the universal cover of $G_{\mathrm{ss}}$, and $T_{\mathrm{sc}}$ be the preimage of $T_{\mathrm{ss}}$ in $G_{\mathrm{sc}}$. Then $T_{\mathrm{sc}}$ is a maximal split torus of $G_{\mathrm{sc}}$. Moreover, it is well known that

$$
G_{k} / T_{k} \cong G_{\mathrm{ss}} / T_{\mathrm{ss}} \cong G_{\mathrm{sc}} / T_{\mathrm{sc}}
$$

and $\operatorname{rank}\left(\operatorname{Pic}\left(G_{\mathrm{sc}} / T_{\mathrm{sc}}\right)\right)=\operatorname{rank}\left(T_{\mathrm{sc}}\right)$. (Here $\operatorname{Pic}(X)$ is the Picard group generated by all line bundles over $X$.) So either $Z$ is trivial (and then $G_{k}$ is semisimple) or $\operatorname{rank}\left(T_{k}\right)>\operatorname{rank}\left(\operatorname{Pic}\left(G_{k} / T_{k}\right)\right)$.

Here, we assume that $G_{k}$ is semisimple, i.e.

$$
\ell=\operatorname{rank}\left(T_{k}\right)=\operatorname{rank}\left(\operatorname{Pic}\left(G_{k} / T_{k}\right)\right)
$$

Let $t_{i} \in \operatorname{Pic}\left(G_{k} / T_{k}^{i}\right)$ be the line bundle corresponding to the $\mathbb{G}_{\mathrm{m}}$-torsor $G_{k} / T_{k}^{i-1} \rightarrow$ $G_{k} / T_{k}^{i}$. Let $E\left(G_{k} / T_{k}^{i}\right)$ be the total space of this bundle so that $G_{k} / T_{k}^{i-1}$ is an open subset of $E\left(G_{k} / T_{k}^{i}\right)$. Then we have maps

$$
Y=G_{k} / T_{k}^{i} \stackrel{g}{\rightarrow} X=E\left(G_{k} / T_{k}^{i}\right) \stackrel{f}{\hookleftarrow} U=X-Y=G_{k} / T_{k}^{i-1}
$$

and the localization exact sequence of Chow groups

$$
\mathrm{CH}^{0}\left(G_{k} / T_{k}^{i}\right) \stackrel{t_{i}}{\rightarrow} \mathrm{CH}^{1}\left(G_{k} / T_{k}^{i}\right) \rightarrow \mathrm{CH}^{1}\left(G_{k} / T_{k}^{i-1}\right) \rightarrow 0
$$


where $\mathrm{CH}^{i}(X)$ is the Chow group of codimension $i$ algebraic cycles modulo rational equivalence.

Hence each $\operatorname{Pic}\left(G_{k} / T_{k}^{i}\right)=\mathrm{CH}^{1}\left(G_{k} / T_{k}^{i}\right)$ is a quotient of $\operatorname{Pic}\left(G_{k} / T_{k}^{\ell}\right)$. So each $t_{i}$ can be written as an element in $\operatorname{Pic}\left(G_{k} / T_{k}^{\ell}\right)$. The Picard group is generated by line bundles corresponding to the characters of the associated simply connected group $G_{\mathrm{sc}}$. Let us write it as

$$
\operatorname{Pic}\left(G_{k} / T_{k}\right)=\mathbb{Z}\left\{s_{1}, \ldots, s_{\ell}\right\} .
$$

Here $\mathbb{Z}\left\{a_{1}, \ldots, a_{n}\right\}$ is a free $\mathbb{Z}$-module generated by $a_{1}, \ldots, a_{n}$. (So if $G$ is simply connected, we can take $t_{i}=s_{i}$.) Thus we have

$$
\operatorname{Pic}\left(G_{k} / T_{k}^{i}\right) \cong \mathbb{Z}\left\{s_{1}, \ldots, s_{\ell}\right\} /\left(t_{i+1}, \ldots, t_{\ell}\right)
$$

where the right hand module is the quotient module of $\mathbb{Z}\left\{s_{1}, \ldots, s_{\ell}\right\}$ by the submodule generated by $t_{i+1}, \ldots, t_{\ell}$. In particular, since $G$ is semisimple, $\operatorname{Pic}\left(G_{k}\right)=$ $\operatorname{Pic}\left(G_{k} / T_{k}^{0}\right)$ is finite (the quotient of the lattice of weights which are characters of $T_{\mathrm{sc}}$ by the lattice of characters of $T$ ).

Now we consider the localization (long) exact sequence for Witt theories

$$
\rightarrow W^{*-1}\left(G_{k} / T_{k}^{i} ; t_{i}+L\right) \stackrel{g_{*}}{\rightarrow} W^{*}\left(G_{k} / T_{k}^{i} ; L\right) \stackrel{f^{*}}{\rightarrow} W^{*}\left(G_{k} / T_{k}^{i-1} ; L\right) \stackrel{\delta}{\rightarrow} \cdots .
$$

Let $B_{k}$ be the Borel subgroup of $G_{k}$ containing $T_{k}$. Then the fiber of the flat map $G_{k} / T_{k} \rightarrow G_{k} / B_{k}$ is the unipotent group $U_{k}$ which is an affine space. Hence by the homotopy invariance of Witt groups [Gi], we have an isomorphism $W^{*}\left(G_{k} / T_{k} ; L\right) \cong W^{*}\left(G_{k} / B_{k} ; L\right)$. We recall the result of Calmès and Fasel:

Lemma 2.1 ([Ca-Fa]). If $L \neq 0 \in \operatorname{Pic}\left(G_{k} / T_{k}\right) / 2$, then $W^{*}\left(G_{k} / B_{k} ; L\right)=0$.

For an algebraically closed field $k$ with $\operatorname{ch}(k)=0$, we will give a topological proof of this fact in $\S 5$.

Lemma 2.2. Let $G$ be semisimple. For $0 \leq i \leq \ell$, if $L$ is a line bundle on $G_{k} / T_{k}$ that stays nontrivial $\bmod (2)$ when pulled back to $G_{k} / T_{k}^{i}$, then $W^{*}\left(G_{k} / T_{k}^{i} ; L\right)=0$.

Proof. Let $L_{\ell-1} \in \operatorname{Pic}\left(G_{k} / T_{k}\right)$ be such that $L_{\ell-1} \neq 0 \bmod \left(2, t_{\ell}\right)$. Consider the localization exact sequence

$\rightarrow W^{*-1}\left(G_{k} / T_{k}^{\ell} ; t_{\ell}+L_{\ell-1}\right) \stackrel{g_{*}}{\longrightarrow} W^{*}\left(G_{k} / T_{k}^{\ell} ; L_{\ell-1}\right) \stackrel{f^{*}}{\longrightarrow} W^{*}\left(G_{k} / T_{k}^{\ell-1} ; L_{\ell-1}\right) \stackrel{\delta}{\rightarrow} \cdots$.

Since the first and second terms are zero from the result by Calmès and Fasel, also $W^{*}\left(G_{k} / T_{k}^{\ell-1}, L_{\ell-1}\right)=0$.

Let $L_{i-1} \in \operatorname{Pic}\left(G_{k} / T_{k}\right)$ such that $L_{i-1} \neq 0 \bmod \left(2, t_{i}, \ldots, t_{\ell}\right)$. Next we consider the localization exact sequence

$\rightarrow W^{*-1}\left(G_{k} / T_{k}^{i} ; t_{i}+L_{i-1}\right) \stackrel{g_{*}}{\longrightarrow} W^{*}\left(G_{k} / T_{k}^{i} ; L_{i-1}\right) \stackrel{f^{*}}{\longrightarrow} W^{*}\left(G_{k} / T_{k}^{i-1} ; L_{i-1}\right) \stackrel{\delta}{\rightarrow} \cdots$. 
By descending induction on $i$, we assume the first and second terms are zero. Then $W^{*}\left(G_{k} / T_{k}^{i-1}, L_{i-1}\right)=0$.

Theorem 2.3. Let $G$ be simply connected. Then $W^{*}\left(G_{k}\right) \cong W^{*}\left(G_{k} / T_{k}\right)$.

Proof. By the assumption, we can take $t_{i}=s_{i}$. Since $G_{k}$ is semisimple, we see $\operatorname{rank}\left(\operatorname{Pic}\left(G_{k} / T_{k}\right)\right)=\ell$. We consider the localization exact sequence, for $1 \leq i \leq \ell$,

$$
\rightarrow W^{*-1}\left(G_{k} / T_{k}^{i} ; t_{i}\right) \stackrel{g_{*}}{\rightarrow} W^{*}\left(G_{k} / T_{k}^{i}\right) \stackrel{f^{*}}{\rightarrow} W^{*}\left(G_{k} / T_{k}^{i-1}\right) \stackrel{\delta}{\rightarrow} \cdots .
$$

Since the first term is zero from Lemma 2.2, we have

$$
W^{*}\left(G_{k} / T_{k}^{i}\right) \cong W^{*}\left(G_{k} / T_{k}^{i-1}\right),
$$

and hence we get the theorem as

$$
W^{*}\left(G_{k}\right) \cong W^{*}\left(G_{k} / T_{k}^{1}\right) \cong \cdots \cong W^{*}\left(G_{k} / T_{k}^{\ell}\right)=W^{*}\left(G_{k} / T_{k}\right) .
$$

Next we consider the case when $G$ is not simply connected.

Corollary 2.4. Let $G_{\mathrm{ss}}=G_{k} / T_{k}^{m}$ for $0 \leq m \leq \ell$ and $\operatorname{Pic}\left(G_{k}\right) \cong(\mathbb{Z} / 2)^{n}$ for $n \geq 0$. Then there is an additive isomorphism

$$
W^{*}\left(G_{k}\right) \cong W^{*}\left(G_{k} / T_{k}\right) \otimes \Lambda\left(x_{1}, \ldots, x_{n+m}\right) \quad \text { with } \quad \operatorname{deg}\left(x_{i}\right)=0 .
$$

Proof. For a sequence $T^{1} \subset \cdots \subset T^{\ell}$ of maximal tori, we take $t_{i} \in \operatorname{Pic}\left(G_{k} / T_{k}\right)$ such that

$t_{i}=0(1 \leq i \leq m), \quad t_{i}=2 s_{i}(m+1 \leq i \leq m+n), \quad t_{i}=s_{i}(m+n+1 \leq i \leq \ell)$.

We consider the decomposition of the universal cover $\left((\mathbb{Z} / 2)^{n}\right.$-cover $) \pi: G_{\mathrm{sc}} \rightarrow$ $G_{\mathrm{ss}}=G_{k} / T_{k}^{m}$ such that

$$
\pi: G_{\mathrm{sc}}=G(n) \stackrel{\pi_{n}}{\longrightarrow} G(n-1) \rightarrow \cdots \stackrel{\pi_{1}}{\longrightarrow} G(0)=G_{\mathrm{ss}}
$$

with $\operatorname{Pic}(G(j)) \cong(\mathbb{Z} / 2)^{n-j}$ (that is, $G(i)$ is the $(\mathbb{Z} / 2)^{i}$-cover of $\left.G_{\mathrm{SS}}\right)$. Take elements $t_{i}(j) \in \operatorname{Pic}(G(j) / T(j))$ for the sequence of tori in $G(j)$ such that

$$
t(j)_{i}=s_{i}=\frac{1}{2} t_{i}(m+1 \leq i \leq m+j), \quad t(j)_{i}=t_{i}(i \leq m \text { or } m+j<i) .
$$

We consider the diagram

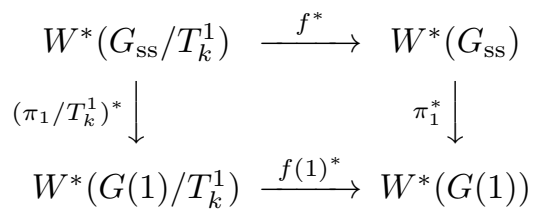


where $f^{*}, f(1)^{*}$ and $\left(\pi_{1} / T_{k}^{1}\right)^{*}$ are pull-backs of the projections

$$
G(1) \stackrel{\pi}{\rightarrow} G_{\mathrm{ss}} \stackrel{f}{\rightarrow} G_{\mathrm{ss}} / T_{k}^{1}, \quad G(1) \stackrel{f(1)}{\longrightarrow} G(1) / T_{k}^{1} \stackrel{\pi_{1} / T_{k}^{1}}{\longrightarrow} G_{\mathrm{ss}} / T_{k}^{1} .
$$

Here $f(1)^{*}$ is an isomorphism, since $W^{*}\left(G(1) / T^{1}, t(1)_{m+1}\right)=0$ from the equality $t(1)_{m+1}=s_{m+1}$. The map $\left(\pi_{1} / T^{1}\right)^{*}$ is also an isomorphism, since $t(1)_{i}=t_{i}$ for $i>m+1$. (Note $G_{\mathrm{ss}} / T_{k}^{1}$ is constructed from $G_{k} / T_{k}^{\ell}$, using $t_{i}$ only for $i>m+1$ but not $i=m+1$.) The commutativity of the above diagram implies that $f^{*}$ is split injective.

Consider the exact sequence

$$
\rightarrow W^{*}\left(G_{\mathrm{ss}} / T_{k}^{1} ; t_{m+1}\right) \stackrel{f^{*}}{\longrightarrow} W^{*}\left(G_{\mathrm{ss}}\right) \stackrel{\delta}{\rightarrow} W^{*}\left(G_{\mathrm{ss}} / T_{k}^{1}\right) \stackrel{g_{*}}{\rightarrow} \cdots .
$$

From the injectivity of $f^{*}$ and $t_{m+1}=2 s_{m+1}$, we have

$$
W^{*}\left(G_{\mathrm{ss}}\right) \cong W^{*}\left(G(1) / T_{k}^{1}\right)\left\{1, x_{1}\right\},
$$

that is, $W^{*}\left(G_{\mathrm{ss}}\right)$ is a free $W^{*}\left(G(1) / T_{k}^{1}\right)$-module generated by 1 and $x_{1}$. Similarly, $W^{*}(G(j)) \cong W^{*}\left(G(j+1) / T_{k}^{1}\right)\left\{1, x_{j+1}\right\}$. Thus we have additive isomorphisms

$$
W^{*}\left(G_{\mathrm{ss}}\right) \cong W^{*}\left(G_{\mathrm{sc}} / T_{k}^{n}\right) \otimes \Lambda\left(x_{1}, \ldots, x_{n}\right) \cong W^{*}\left(G_{\mathrm{sc}} / T_{\mathrm{sc}}\right) \otimes \Lambda\left(x_{1}, \ldots, x_{n}\right) .
$$

Next, we consider the case of $m>0$. Recall $G_{\mathrm{ss}}=G_{k} / T_{k}^{m}$. We consider the localization exact sequence

$$
\rightarrow W^{*-1}\left(G_{\mathrm{ss}} ; t_{m}\right) \stackrel{g_{*}}{\rightarrow} W^{*}\left(G_{\mathrm{ss}}\right) \stackrel{f^{*}}{\rightarrow} W^{*}\left(G_{k} / T_{k}^{m-1}\right) \stackrel{\delta}{\rightarrow} \cdots .
$$

Here $t_{m}=0 \in \operatorname{Pic}\left(G_{\mathrm{ss}}\right)$. So the normal bundle for $G_{\mathrm{ss}} \subset E\left(G_{\mathrm{ss}}\right)$ is trivial. Hence from Lemma 2.5 below, the Gysin map $g_{*}$ is trivial and the resulting short exact sequence splits. Then we get the desired result

$$
W^{*}\left(G_{k} / T_{k}^{m-1}\right) \cong W^{*}\left(G_{\mathrm{ss}}\right)\left\{1, x_{n+1}\right\} \quad \text { with } \quad \operatorname{deg}\left(x_{1}\right)=0 .
$$

Similarly,

$$
W^{*}\left(G_{k}\right) \cong W^{*}\left(G_{\mathrm{ss}}\right) \otimes \Lambda\left(x_{n+1}, \ldots, x_{n+m}\right) \quad \text { with } \quad \operatorname{deg}\left(x_{i}\right)=0 .
$$

Thus we have the corollary.

The Balmer-Witt group can be extended as a generalized cohomology theory for the $\mathbb{A}^{1}$-homotopy category ([Ho], [Sch], [Sch-Tr]; see $\S 5$ ). For smooth $X$ with a line bundle $L$, let $\operatorname{Th}(L)$ be its Thom space, which is an object of the $\mathbb{A}^{1}$-homotopy category. We note that the Witt group $W^{*}(X ; L)$ can be written by using the Thom space $\operatorname{Th}(L)([\mathrm{Zi}])$, namely, $W^{*+1}(X ; L) \cong \tilde{W}^{*}(\operatorname{Th}(L))$ where $\tilde{W}^{*}(-)$ is the reduced Witt theory. 
Let $t \in \operatorname{Pic}(X)$ and let $E(t)$ (resp. $T(t))$ be the total space of the line bundle $t$ (resp. the $\mathbb{G}_{\mathrm{m}}$-torsor induced from $t$ ). Then from the cofiber sequence $T(t) \stackrel{f}{\subset}$ $E(t) \stackrel{q}{\rightarrow} \mathrm{Th}(t)$ (where $q$ is the projection), in the $\mathbb{A}^{1}$-homotopy category we have the long exact sequence (see $\S 5$ below)

$$
\rightarrow \tilde{W}^{*}(\operatorname{Th}(t)) \stackrel{q^{*}}{\rightarrow} W^{*}(E(t)) \stackrel{f^{*}}{\rightarrow} W^{*}(T(t)) \stackrel{\delta}{\rightarrow} \cdots
$$

Here $\tilde{W}^{*}(\operatorname{Th}(t)) \cong W^{*}(X ; t)$, and the map $q^{*}$ corresponds to the Gysin map $g_{*}$.

Lemma 2.5. If $t=0 \in \operatorname{Pic}(X)$, then $q^{*}=0$ (hence $g_{*}=0$ ) and we have $a$ $W^{*}(X)$-module isomorphism

$$
W^{*}(T(t)) \cong W^{*}(X)\left\{1, x_{0}\right\} \text { with } \operatorname{deg}\left(x_{0}\right)=0 \text {. }
$$

Proof. Since $t$ is trivial, we see $E(t)=X \times \mathbb{A}, T(t)=X \times(\mathbb{A}-\{0\})$, and $\operatorname{Th}(t)=$ $X \times(\mathbb{A} /(\mathbb{A}-\{0\}))$. We consider the long exact sequence induced from the trivial line bundle over $\{0\}$

$$
\rightarrow \tilde{W}^{*}(\mathbb{A} /(\mathbb{A}-\{0\})) \stackrel{q^{*}}{\rightarrow} W^{*}(\mathbb{A}) \stackrel{f^{*}}{\rightarrow} W^{*}(\mathbb{A}-\{0\}) \stackrel{\delta}{\rightarrow} \cdots .
$$

Here $f^{*}$ is a split injection, since $k^{*} f^{*}: W^{*}(\mathbb{A}) \rightarrow W^{*}(\mathrm{pt})$ is an isomorphism for maps pt $\stackrel{k}{\hookrightarrow} \mathbb{A}-\{0\} \stackrel{f}{\hookrightarrow} \mathbb{A}$. Hence the above exact sequence splits as

$$
\begin{aligned}
W^{*}(\mathbb{A}-\{0\}) & \cong W^{*}(\mathbb{A}) \oplus \tilde{W}^{*-1}(\mathbb{A} /(\mathbb{A}-\{0\})) \\
& \cong W^{*}(\mathrm{pt})\left\{1, x_{0}\right\} \text { with } x_{0}=\delta^{-1}(\sigma) .
\end{aligned}
$$

Here $W^{*}(\mathbb{A} /(\mathbb{A}-\{0\})) \cong W^{*}\left(S^{2,1}\right) \cong W^{*}(\mathrm{pt})\{1, \sigma\}$ with $\operatorname{deg}(\sigma)=1$. Since $W^{*}(X)$ has a good (external) product $[\mathrm{Pa}-\mathrm{Wa}]$, we have $W^{*}(T(t)) \cong W^{*}(X)\left\{1, x_{0}\right\}$.

\section{$\S 3$. Cohomology theories of compact Lie groups $G$}

Let $G$ be a compact simply connected Lie group (hence $G$ is semisimple so that $\pi_{1}(G)$ is finite). By the Borel theorem (see [Ka]), we have a ring isomorphism

$$
H^{*}(G ; \mathbb{Z} / 2) \cong P^{\prime}(x) \quad \text { with } \quad P^{\prime}(x)=\bigotimes_{i} \mathbb{Z} / 2\left[x_{j}\right] /\left(x_{j}^{2^{s_{j}}}\right)
$$

where the (cohomological) degrees $\left|x_{j}\right|$ are odd. When $s^{i} \neq 0$, let us write $y_{i}=x_{i}^{2}$ so that

$$
H^{*}(G ; \mathbb{Z} / 2) \cong P(y) \otimes \Delta\left(x_{1}, \ldots, x_{l}\right) \quad \text { with } \quad P(y)=\bigotimes_{i} \mathbb{Z} / 2\left[y_{i}\right] /\left(y_{i}^{2^{r_{i}}}\right)
$$


where $r_{i}=s_{i}-1$ and $A \otimes \Delta\left(x_{1}, \ldots, x_{\ell}\right)$ is additively an $A$-free module generated by $x_{j_{1}} \ldots x_{j_{k}}$ for all $1 \leq j_{1}<\cdots<j_{k} \leq \ell$, and

$$
x_{j}^{2}=\sum_{j_{1}<\cdots<j_{k}} a_{j_{1}, \ldots, j_{k}} x_{j_{1}} \ldots x_{j_{k}}, \quad a_{j_{1}, \ldots, j_{k}} \in A^{+} .
$$

Let $T$ be a maximal torus of $G$ and $B T$ the classifying space of $T$. We consider the fiber bundle ([Tod]) $G \stackrel{\pi}{\rightarrow} G / T \stackrel{i}{\rightarrow} B T$ and the induced spectral sequence

$$
E_{2}^{*, *^{\prime}}=H^{*}\left(B T ; H^{*^{\prime}}(G ; \mathbb{Z} / 2)\right) \Rightarrow H^{*+*^{\prime}}(G / T ; \mathbb{Z} / 2) .
$$

The cohomology of the classifying space of the torus is

$$
H^{*}(B T ; \mathbb{Z} / 2) \cong S(t)=\mathbb{Z} / 2\left[t_{1}, \ldots, t_{\ell}\right] \quad \text { with } \quad\left|t_{i}\right|=2,
$$

where $\ell$ is also the number of odd degree generators $x_{i}$ in $H^{*}(G ; \mathbb{Z} / 2)$. (We are assuming that $G$ is simply connected, and $t_{i}=s_{i}$ in the notation of $\S 2$.)

For dimensional reasons, each $t_{i} \in E_{2}^{2,0}$ is a permanent cycle (i.e. $0 \neq t_{i}$ $\left.\in E_{\infty}^{*, *^{\prime}}\right)$. It is also known that $y_{i}$ is a permanent cycle and that there is a regular sequence ([Tod], [Mi-Ni]) $\left(b_{1}, \ldots, b_{\ell}\right)$ in $H^{*}(B T ; \mathbb{Z} / 2)$ such that $d_{\left|x_{i}\right|+1}\left(x_{i}\right)=b_{i}$. Thus we get by induction (for $\left|x_{1}\right| \leq \cdots \leq\left|x_{\ell}\right|$ )

$$
E_{\left|x_{\ell}\right|+2}^{*, *^{\prime}} \cong P(y) \otimes S(t) /\left(b_{1}, \ldots, b_{\ell}\right) .
$$

Here elements in $P(y) \otimes S(t)$ are cycles with $d_{r}=0$ for all $r$, which are generated by even-dimensional elements. Hence $E_{\infty}^{*, *^{\prime}} \cong E_{\left|x_{\ell}\right|+2}^{*, *^{\prime}}$.

Let $T^{1} \subset \cdots \subset T^{\ell}=T$ be a sequence of tori of $G$ where $T^{i} \cong\left(S^{1}\right)^{\times i}$. The $S^{1}$-fiber bundle $S^{1} \rightarrow G / T^{i-1} \rightarrow G / T^{i}$ induces the Gysin exact sequence

$$
\stackrel{\delta}{\rightarrow} H^{*-2}\left(G / T^{i} ; \mathbb{Z} / 2\right) \stackrel{j_{*}=\times t_{i}}{\longrightarrow} H^{*}\left(G / T^{i} ; \mathbb{Z} / 2\right) \rightarrow H^{*}\left(G / T^{i-1} ; \mathbb{Z} / 2\right) \stackrel{\delta}{\rightarrow} \cdots .
$$

Using this argument, we can compute $H^{*}\left(G / T^{i} ; \mathbb{Z} / 2\right)$ from $H^{*}\left(G / T^{\ell} ; \mathbb{Z} / 2\right)$.

Lemma 3.1. Let $G$ be a (connected) simple Lie group. Then we can take generators $t_{1}, \ldots, t_{\ell}$ in $S(t)$ such that the sequence $b_{1}, \ldots, b_{i}$ is regular in $S(t) /\left(t_{i+1}, \ldots, t_{\ell}\right)$, and

$$
b_{i}=t_{i} g_{i} \quad \text { in } S(t) /\left(b_{1}, \ldots, b_{i-1}, t_{i+1}, \ldots, t_{\ell}\right)
$$

for some $g_{i} \in S(t)$.

Proof. From [Ya2, §4], we know that the above fact holds for each simple Lie group $G$ (with $p=2$ in [Ya2, Lemma 2.1]) except for $G=\operatorname{Sp}(n), E_{8}$. (Note $H^{*}(\operatorname{Spin}(n) / T ; \mathbb{Z} / 2) \cong H^{*}(S O(n) / T ; \mathbb{Z} / 2)$.) The case $G=\operatorname{Sp}(n)$ is almost immediate since

$$
H^{*}(G / T ; \mathbb{Z} / 2) \cong \mathbb{Z} / 2\left[t_{1}, \ldots, t_{n}\right] /\left(c_{1}^{2}, \ldots, c_{n}^{2}\right),
$$


where $c_{i}$ is the $i$-th elementary symmetric function in $t_{1}, \ldots, t_{n}$. In fact the sequence $c_{1}^{2}, \ldots, c_{n}^{2}$ is regular and $c_{i}^{2}=g_{i} t_{i} \bmod \left(t_{i+1}, \ldots, t_{n}\right)$.

For $G=E_{8}$, we use a result by Kono-Ishitoya [Ko-Is] and Ohsita [Oh]. It is known that gr $H^{*}(G / T ; \mathbb{Z} / 2) /(P(y))$ is isomorphic to

$$
S(t) /\left(b_{1}, \ldots, b_{8}\right) \cong S(t) /\left(c_{2}, c_{3}, c_{5}^{\prime}, c_{9}^{\prime}, I_{8}, I_{12}, I_{14}, I_{15}\right)
$$

in the notation of [Ko-Is] (see [Ko-Is] for details). Here $c_{9}^{\prime}=c_{1}\left(c_{8}+c_{7} c_{1}+c_{0} c_{1}^{2}\right)$ and we put $b_{8}^{\prime}=c_{9}^{\prime}$ and $t_{8}^{\prime}=c_{1}$. Next we see $I_{15}=c_{8} c_{7} \bmod \left(c_{1}\right)$, and hence put $t_{7}^{\prime}=t_{8}$. We have

$$
I_{14}=c_{7}^{2}, I_{12}=c_{6}^{2}, c_{5}^{\prime}=c_{5}, I_{8}=c_{4}^{2} \bmod \left(c_{1}, c_{8}\right) .
$$

Then we can take $t_{i}^{\prime}, b_{i}^{\prime}$ such that $\operatorname{Ideal}\left(b_{1}^{\prime}, \ldots, b_{8}^{\prime}\right)=\operatorname{Ideal}\left(b_{1}, \ldots, b_{8}\right)$ and the elements $t_{i}^{\prime}, b_{i}^{\prime}$ satisfy the condition in this lemma for $G=E_{8}$.

Using this lemma, we can prove Corollary 3.2, Theorem 3.4 and Corollary 3.5 below (see also [Ya2]).

Corollary 3.2 ([Ya2, Lemma 2.1]). Let $G$ be a compact simply connected simple Lie group. Then for $0 \leq i \leq \ell$ we have an isomorphism

$$
H^{*}\left(G / T^{i} ; \mathbb{Z} / 2\right) \cong H^{*}(G / T) /\left(t_{i+1}, \ldots, t_{\ell}\right) \otimes \Delta\left(x_{i+1}^{\prime}, \ldots, x_{\ell}^{\prime}\right),
$$

where $f_{i}^{*}\left(x_{j}^{\prime}\right)=x_{j}$ for the projection $f_{i}: G \rightarrow G / T^{i}$.

When $G=S O(n)$, we know $\operatorname{Pic}\left(G_{k}\right) \cong \mathbb{Z} / 2$ and we can take (with the notation of $\S 2) t_{1}=2 s_{1}$ and $t_{i}=s_{i}$ for $i>1$. Hence we can easily prove the following corollary, by using similar arguments.

Corollary 3.3. Let $G=S O(n)$. Then the isomorphism of Corollary 3.2 holds for $i \geq 1$. For $i=0$, we have

$$
H^{*}\left(G_{k} ; \mathbb{Z} / 2\right) \cong H^{*}(G / T) /\left(t_{2}, \ldots, t_{\ell}\right) \otimes \Delta\left(x_{1}, \ldots, x_{\ell}\right) .
$$

Examples. When $G=S U(n)$, we know that $P(y) \cong \mathbb{Z} / 2$ and

$$
\begin{aligned}
H^{*}(G ; \mathbb{Z} / 2) & \cong \Lambda\left(x_{3}, x_{5}, \ldots, x_{2 n-1}\right) \quad \text { with } \quad\left|x_{j}\right|=j, \\
H^{*}(G / T ; \mathbb{Z} / 2) & \cong \mathbb{Z} / 2\left[t_{1}, \ldots, t_{n}\right] /\left(c_{1}, \ldots, c_{n}\right) .
\end{aligned}
$$

Of course $b_{i}=c_{i}$ is regular and satisfies (with $g_{i}=t_{1} \ldots t_{i-1}$ ) the conclusion of Lemma 3.1. Hence

$$
H^{*}\left(G / T^{i} ; \mathbb{Z} / 2\right) \cong \mathbb{Z} / 2\left[t_{1}, \ldots, t_{i}\right] /\left(c_{1}, \ldots, c_{i}\right) \otimes \Lambda\left(x_{2 i-1}^{\prime}, \ldots, x_{2 n-1}^{\prime}\right) .
$$


Let $X$ be an algebraic variety over $k \subset \mathbb{C}$. Let $H^{*, *^{\prime}}(X ; \mathbb{Z} / 2)$ be the $\bmod (2)$ motivic cohomology over $k$ constructed by Suslin and Voevodsky [Vo1, Vo2]. For every nonzero element $x \in H^{m, n}(X ; \mathbb{Z} / 2)$, we define the weight degree and the difference degree by

$$
w(x)=2 n-m, \quad d(x)=m-n .
$$

When $X$ is smooth, it is known that $w(x) \geq 0$ and $d(x) \leq \operatorname{dim}(X)$ for $x \neq 0$ (for example see [Vo1, Corollary 2.3].) Moreover the affirmative answer by Voevodsky to the Milnor conjecture (and hence the Beilinson-Lichtenbaum conjecture) implies

$$
H^{*, *^{\prime}}(\mathrm{pt} ; \mathbb{Z} / 2) \cong \mathbb{Z} / 2[\tau] \otimes K_{*}^{M}(k)
$$

where $0 \neq \tau \in H^{0,1}(\mathrm{pt} ; \mathbb{Z} / 2) \cong \mathbb{Z} / 2$ and the $\bmod (2)$ Milnor $K$-theory $K_{*}^{M}(k) / 2$ is isomorphic to $H^{*, *}(\mathrm{pt} ; \mathbb{Z} / 2)$.

Let us denote by $G_{k}$ the split reductive group over $k$ corresponding to the compact connected simple Lie group $G$, and $T_{k}$ the split torus. It is well known that $G_{k} / B_{k}$ is cellular. The fiber of the projection $G_{k} / T_{k} \rightarrow G_{k} / B_{k}$ is a unipotent group. By the homotopy invariance of motivic cohomology, we have $H^{*, *^{\prime}}\left(G_{k} / B_{k} ; \mathbb{Z} / 2\right) \cong$ $H^{*, *^{\prime}}\left(G_{k} / T_{k} ; \mathbb{Z} / 2\right)$. Let $G_{\mathbb{C}} / T_{\mathbb{C}}$ be the flag variety for $k=\mathbb{C}$. Then

$$
\begin{aligned}
& H^{*, *^{\prime}}\left(G_{k} / T_{k} ; \mathbb{Z} / 2\right) \cong K_{*}^{M}(k) / 2 \otimes H^{*, *^{\prime}}\left(G_{\mathbb{C}} / T_{\mathbb{C}} ; \mathbb{Z} / 2\right) \\
& \quad \cong H^{*, *^{\prime}}(\mathrm{pt} ; \mathbb{Z} / 2) \otimes H^{*}(G / T ; \mathbb{Z} / 2) \text { with } w\left(H^{*}(G / T ; \mathbb{Z} / 2)\right)=0 .
\end{aligned}
$$

In particular, the base change (or the realization) map $t_{\mathbb{C}}: G_{k} / T_{k} \rightarrow G_{\mathbb{C}} / T_{\mathbb{C}}$ induces an isomorphism $\operatorname{Pic}\left(G_{k} / T_{k}\right) / 2 \cong \operatorname{Pic}\left(G_{\mathbb{C}} / T_{\mathbb{C}}\right) / 2$, since $\operatorname{Pic}(X) / 2 \cong \mathrm{CH}^{1}(X) / 2 \cong$ $H^{2,1}(X ; \mathbb{Z} / 2)$ and

$$
H^{2,1}\left(G_{k} / T_{k} ; \mathbb{Z} / 2\right) \cong H^{2,1}\left(G_{\mathbb{C}} / T_{\mathbb{C}} ; \mathbb{Z} / 2\right) \cong H^{2}(G / T ; \mathbb{Z} / 2) .
$$

For the motivic theory, we also have the Thom isomorphism and hence the Gysin exact sequence ([Vo1, Vo3])

$\stackrel{\delta}{\rightarrow} H^{*-2, *^{\prime}-1}\left(G_{k} / T_{k}^{i} ; \mathbb{Z} / 2\right) \stackrel{\times t_{i}}{\longrightarrow} H^{*, *^{\prime}}\left(G_{k} / T_{k}^{i} ; \mathbb{Z} / 2\right) \rightarrow H^{*, *^{\prime}}\left(G_{k} / T_{k}^{i-1} ; \mathbb{Z} / 2\right) \stackrel{\delta}{\rightarrow} \cdots$.

Since $H^{2 *+1, *}\left(G_{k} / T_{k}^{i} ; \mathbb{Z} / 2\right)=0$ (for the negative weight degree), we have

$$
H^{2 *-2, *-1}\left(G_{k} / T_{k}^{i} ; \mathbb{Z} / 2\right) \stackrel{\times t_{i}}{\longrightarrow} H^{2 *, *}\left(G_{k} / T_{k}^{i} ; \mathbb{Z} / 2\right) \rightarrow H^{2 *, *}\left(G_{k} / T_{k}^{i-1} ; \mathbb{Z} / 2\right) \stackrel{\delta}{\rightarrow} 0
$$

for $i \geq 1$ when $G$ is simply connected (for $i \geq 2$ when $G=S O(n)$ ). By descending induction on $i$, we easily show

$$
H^{2 *, *}\left(G_{k} / T^{i} ; \mathbb{Z} / 2\right) \cong H^{2 *}(G / T ; \mathbb{Z} / 2) /\left(t_{i+1}, \ldots, t_{\ell}\right)
$$

and there is $x_{i} \in H^{2 *-1, *}\left(G_{k} / T^{i-1} ; \mathbb{Z} / 2\right)$ with $\delta\left(x_{i}\right)=b_{i}$. Moreover, we have the following theorems: 
Theorem 3.4 ([Ya2, Theorem 3.1]). Let $G$ be a compact connected simple Lie group. Then for $0 \leq i \leq \ell$ there are $H^{*, *^{\prime}}(\mathrm{pt} ; \mathbb{Z} / 2)$-module isomorphisms

$$
\begin{aligned}
H^{*, *^{\prime}}\left(G_{k} / T_{k}^{i} ; \mathbb{Z} / 2\right) & \cong H^{*}\left(G / T^{i} ; \mathbb{Z} / 2\right) \otimes H^{*, *^{\prime}}(\mathrm{pt} ; \mathbb{Z} / 2) \\
& \cong H^{*}(G / T) /\left(t_{i+1}, \ldots, t_{\ell}\right) \otimes \Lambda\left(x_{i+1}^{\prime}, \ldots, x_{\ell}^{\prime}\right) \otimes H^{*, *^{\prime}}(\mathrm{pt} ; \mathbb{Z} / 2)
\end{aligned}
$$

where the bidegree is given by $w\left(x_{i}^{\prime}\right)=1$ and $w(u)=0$ for $u \in H^{*}(G / T ; \mathbb{Z} / 2)$, i.e. $\operatorname{deg}\left(x_{i}^{\prime}\right)=\left(\left|x_{i}\right|, \frac{1}{2}\left(\left|x_{i}\right|+1\right)\right), \operatorname{deg}(u)=\left(|u|, \frac{1}{2}|u|\right)$.

Corollary 3.5 ([Ya2, Corollary 3.2]). There is an $H^{*, *^{\prime}}(\mathrm{pt} ; \mathbb{Z} / 2)$-algebra isomorphism

$$
H^{*, *^{\prime}}\left(G_{k} ; \mathbb{Z} / 2\right) \cong H^{*, *^{\prime}}(\mathrm{pt} ; \mathbb{Z} / 2) \otimes P(y) \otimes \Delta\left(x_{1}, \ldots, x_{\ell}\right)
$$

where $w(P(y))=0$ and $w\left(x_{i}\right)=1$.

Proof. Since $t_{\mathbb{C}}: H^{2 *, *}\left(G_{k} ; \mathbb{Z} / 2\right) \rightarrow H^{2 *}(G ; \mathbb{Z} / 2)$ is injective, we have $y_{i}^{2^{r_{i}}}=0 \in$ $H^{2 *, *}\left(G_{k} ; \mathbb{Z} / 2\right)$. Since $w\left(x_{i}^{2}\right)=2, t_{\mathbb{C}}(\tau)=1$ and $K_{+}^{M}(k) \subset \operatorname{Ker}\left(t_{\mathbb{C}}\right)$, we see $x_{i}^{2}=\tau y_{j}$ modulo the ideal generated by $K_{+}^{M}(k) / 2$.

Theorem 3.6 ([Ya2, Theorem 3.3]). Let $G$ be a compact connected simple Lie group. Suppose that $H^{*, *^{\prime}}(X ; \mathbb{Z} / 2)$ is $\mathbb{Z} / 2[\tau]$-free. Then

$$
H^{*, *^{\prime}}\left(X \times G_{k} / T_{k}^{i} ; \mathbb{Z} / 2\right) \cong H^{*, *^{\prime}}(X ; \mathbb{Z} / 2) \otimes_{H^{* \cdot *^{\prime}}(\mathrm{pt} ; \mathbb{Z} / 2)} H^{*, *^{\prime}}\left(G_{k} / T_{k}^{i} ; \mathbb{Z} / 2\right) .
$$

Corollary 3.7 ([Ya2, Corollary 3.4]). Let $G$ be a direct product of compact connected simple Lie groups (e.g. simply connected compact Lie groups). The Künneth formula holds for $H^{*, *^{\prime}}\left(G_{k} ; \mathbb{Z} / 2\right)$, i.e. if $G=G_{1} \times \cdots \times G_{s}$, then

$$
H^{*, *^{\prime}}\left(G_{k} ; \mathbb{Z} / 2\right) \cong H^{*, *^{\prime}}\left(\left(G_{1}\right)_{k} ; \mathbb{Z} / 2\right) \otimes_{\mathbb{Z} / 2[\tau]} \cdots \otimes_{\mathbb{Z} / 2[\tau]} H^{*, *^{\prime}}\left(\left(G_{s}\right)_{k} ; \mathbb{Z} / 2\right) .
$$

In particular $H^{*, *^{\prime}}\left(G_{k} ; \mathbb{Z} / 2\right)$ is a Hopf algebra.

Proof. The conclusion holds for a product of two simple Lie groups, by the above theorem. In the general case, we use induction on $s$.

\section{§4. $K O$-theory}

We explain the $K O$-theory of flag manifolds $G / T$ according to Hara, Kishimoto, Kono and Ohsita ([Ha], $[\mathrm{Ko}-\mathrm{Ha}],[\mathrm{Ki}-\mathrm{Ko}-\mathrm{Oh}],[\mathrm{Ki}-\mathrm{Oh}])$. Recall that the coefficient rings of the (topological) $K O^{*}$-theory and $K U^{*}$-theory are (see [At] or [Ha, §1])

$$
K O^{*} \cong \mathbb{Z}\left[\mu, \mu^{-1}, \eta, w\right] /\left(2 \eta, \eta^{3}, w^{2}-4 \mu, \eta w\right), \quad K U^{*} \cong \mathbb{Z}\left[\beta, \beta^{-1}\right]
$$


with $|\mu|=-8,|w|=-4,|\eta|=-1$ and $|\beta|=-2$. To compute $K O^{*}(G / T)$, we consider the Atiyah-Hirzebruch spectral sequence

$$
E_{2}^{*, *^{\prime}} \cong H^{*}\left(G / T ; K O^{*^{\prime}}\right) \cong H^{*}(G / T) \otimes K O^{*^{\prime}} \Rightarrow K O^{*+*^{\prime}}(G / T) .
$$

It is well known that the first differential is ([Ha, (3.1)], [Ko-Ha], [Fu])

$$
d_{2}(x \otimes \lambda)=\operatorname{Sq}^{2}(\bar{x}) \otimes \lambda \eta, \quad \lambda \in K O^{*},
$$

where $\bar{x} \in H^{*}(G / T ; \mathbb{Z} / 2)$ is the $\bmod (2)$ reduction of $x$.

Note $\mathrm{Sq}^{2} \mathrm{Sq}^{2}=\mathrm{Sq}^{3} \mathrm{Sq}^{1}$ from the Adem relation. So $\operatorname{Sq}^{2} \operatorname{Sq}^{2}(x)=0$ on $H^{*}(G / T ; \mathbb{Z} / 2)$ (since $\left.H^{\text {odd }}(G / T ; \mathbb{Z} / 2)=0\right)$. Let us write simply

$$
H^{*}\left(G / T ; \mathrm{Sq}^{2}\right)=H\left(H^{2 *}(G / T ; \mathbb{Z} / 2) ; \mathrm{Sq}^{2}\right)
$$

for the homology with the differential $\mathrm{Sq}^{2}$. To be compatible with $W^{*}(-)$, we define the degree of $x \in H^{*}\left(G / T ; \mathrm{Sq}^{2}\right)$ to be half that in $H^{*}(G / T ; \mathbb{Z} / 2)$, i.e. $\operatorname{deg}(x)=\frac{1}{2}|x|$. With this convention, we have

$$
E_{3}^{2 *, \text { odd }} \cong E_{3}^{2 *, 8 *^{\prime}-1} \cong H^{*}\left(G / T ; \mathrm{Sq}^{2}\right) \otimes \mathbb{Z} / 2\left[\mu, \mu^{-1}\right]\{\eta\} .
$$

Therefore $E_{\infty}^{2 *,-1}$ is isomorphic to a subquotient of $H^{*}\left(G / T ; \mathrm{Sq}^{2}\right)\{\eta\}$.

Kishimoto, Kono and Ohsita ([Ki-Ko-Oh], [Ki-Oh]) get this homology for $G=$ $U(n), \operatorname{Sp}(n), S O(n), G_{2}, F_{4}, E_{6}$. For example

$$
H^{*}\left(U(2 m+1) / T ; \mathrm{Sq}^{2}\right) \cong \Lambda\left(z_{3}, z_{7}, \ldots, z_{4 m-1}\right)
$$

where $z_{4 s-1}=\sum_{i_{1}<\cdots<i_{s}} t_{i_{1}} t_{i_{2}}^{2} \ldots t_{i_{s}}^{2}$ in $H^{*}(U(2 m+1) / T ; \mathbb{Z} / 2)$, and $\operatorname{Sq}^{2}\left(z_{4 s-1}\right)=0$ (recall $\left.G / T \cong G_{\mathrm{sc}} / T_{\mathrm{sc}}\right)$.

Moreover, Kishimoto, Kono and Ohsita proved that the following assumption is satisfied for all the above groups $G$.

Assumption 4.1. The Atiyah-Hirzebruch spectral sequence for $K O^{*}(G / T)$ collapses from the $E_{3}$-term.

Let $Y$ be a topological space (e.g. a finite-dimensional $C W$-complex). We have the following well known (Bott) exact sequence $((1.1)$ in $[\mathrm{Ha}],(3.4)$ in $[\mathrm{At}])$

$$
\rightarrow K O^{*+1}(Y) \stackrel{\times \eta}{\longrightarrow} K O^{*}(Y) \stackrel{c}{\rightarrow} K U^{*}(Y) \stackrel{r \cdot \beta^{-1}}{\longrightarrow} K O^{*+2}(Y) \rightarrow \cdots,
$$

where $c$ is the complexification map, $r$ is the real restriction map and $\beta$ is the Bott periodicity, that is, the map $\beta^{-1}: K U^{*}(Y) \rightarrow K U^{*+2}(Y)$ is an isomorphism. Let us write

$$
K O^{*}(Y) / K U^{*}(Y)=K O^{*}(Y) /\left(r K U^{*}(Y)\right)=K O^{*}(Y) /\left(r \beta^{-1} K U^{*-2}(Y)\right) .
$$


Then

$$
K O^{*}(Y) / K U^{*}(Y) \cong K O^{*}(Y) /(\operatorname{Ker}(\times \eta)) \cong \operatorname{Im}(\times \eta)\left(K O^{*+1}(Y)\right)
$$

(which is 2 -torsion since so is $\eta$ ). In particular, $K O^{*} / K U^{*} \cong \mathbb{Z} / 2\left[\mu, \mu^{-1}\right]\{1, \eta\}$.

For the rest of this paper, let us write

$$
K O^{2 *}(Y) / K U^{2 *}(Y)=\bigoplus_{r \in \mathbb{Z} / 4} K O^{2 r}(Y) / K U^{2 r}(Y)
$$

(while $K O^{*}(Y)$ means usually $\bigoplus_{r \in \mathbb{Z}} K O^{r}(Y)$ ). We also take the degree modulo 4 in $H^{*}\left(G / T\right.$; $\left.\mathrm{Sq}^{2}\right)$. From (4.1), we see that $\operatorname{Im}(\times \eta)\left(K O^{2 *}(G / T)\right)$ is a subquotient of $H^{*}\left(G / T ; \mathrm{Sq}^{2}\right)$. With this convention, we have

Corollary 4.2. The graded ring $K^{2 *}(G / T) / K U^{2 *}(G / T)$ is isomorphic to a subquotient of $H^{*}\left(G / T ; \mathrm{Sq}^{2}\right)$. Moreover if Assumption 4.1 is satisfied, then

$$
K O^{2 *}(G / T) / K U^{2 *}(G / T) \cong H^{*}\left(G / T ; \mathrm{Sq}^{2}\right)
$$

Let $A, B$ be $\mathbb{Z} / 2$-algebras with differentials $d_{1}$ and $d_{2}$. Then $A \otimes B$ has the differential $d$ given by $d(a \otimes b)=d_{1}(a) \otimes b+a \otimes d_{2}(b)$ for $a \in A, b \in B$. Then it is well known (Künneth formula) that

$$
H(A \otimes B ; d)=H\left(A ; d_{1}\right) \otimes H\left(B ; d_{2}\right)
$$

(which is proved directly from the definition of homology). In particular, for $X, Y$ with $\mathrm{Sq}^{1}=0$, we have

$$
\begin{aligned}
H\left(X \times Y ; \mathrm{Sq}^{2}\right) & =H\left(H^{*}(X \times Y ; \mathbb{Z} / 2) ; \mathrm{Sq}^{2}\right) \\
& \cong H\left(H^{*}(X ; \mathbb{Z} / 2) \otimes H^{*}(Y ; \mathbb{Z} / 2) ; \mathrm{Sq}^{2}\right) \cong H\left(X ; \mathrm{Sq}^{2}\right) \otimes H^{*}\left(Y ; \mathrm{Sq}^{2}\right)
\end{aligned}
$$

For the rest of this section, we assume that $k$ is an algebraically closed subfield of $\mathbb{C}$. When $X$ is a cellular variety over $\mathbb{C}$ (for the definition, see $\S 5$ ), Zibrowius [Zi] shows that

$$
W^{*}(X) \cong K O^{2 *}(X(\mathbb{C})) / K U^{2 *}(X(\mathbb{C})) .
$$

(For another proof, see $\S 5$ below.) This fact can be easily extended to any algebraically closed subfield of $\mathbb{C}$. Hence

Corollary 4.3. Let $G$ be a compact connected Lie group. Suppose that Assumption 4.1 holds. Then the Künneth formula holds for $K O^{*}(G / T) / K U^{*}(G / T)$. Hence from Corollary 3.7,

$$
W^{*}\left(G_{k}\right) \cong H^{*}\left(G / T ; \mathrm{Sq}^{2}\right)
$$

is a Hopf algebra (for an algebraically closed subfield $k$ of $\mathbb{C}$ ). 
Note that the map

$$
q^{*}: W^{*}(X) \rightarrow K O^{2 *}(X(\mathbb{C})) / K U^{2 *}(X(\mathbb{C}))
$$

is an isomorphism for $X=G_{k} / T_{k}$ but is not an isomorphism for $X=G_{k}$, in general.

Example. Let $G=S U(3)$. Then $H^{*}(G ; \mathbb{Z} / 2) \cong \Lambda\left(x_{3}, x_{5}\right)$ and

$$
H^{*}(G / T ; \mathbb{Z} / 2) \cong \mathbb{Z} / 2\left[t_{1}, t_{2}, t_{3}\right] /\left(c_{1}^{\prime}, c_{2}^{\prime}, c_{3}^{\prime}\right) \cong \mathbb{Z} / 2\left[t_{1}, t_{2}\right] /\left(c_{1}^{2}+c_{2}, c_{1} c_{2}\right)
$$

where $c_{i}^{\prime}$ (resp. $c_{i}$ ) is the $i$-th elementary symmetric function of three variables (resp. two variables). Hence

$$
W^{*}\left(G_{k} / T_{k}\right) \cong K O^{2 *}(G / T) / K U^{2 *}(G / T) \cong H^{*}\left(G / T ; \mathrm{Sq}^{2}\right) \cong \Lambda\left(z_{3}\right)
$$

where $z_{3}=t_{1} t_{2}^{2} \cdot\left(\mathrm{Sq}^{2}\left(z_{3}\right)=t_{1}^{2} t_{2}^{2}=c_{2}^{2}=0\right.$.) Then $W^{*}\left(G_{k}\right) \cong W^{*}\left(G_{k} / T_{k}\right)$ and it is a primitive Hopf algebra $\Lambda\left(z_{3}\right)$. On the other hand, we consider the AtiyahHirzebruch spectral sequence converging to $K O^{*}(G)$. Since $\operatorname{Sq}^{2}\left(x_{3}\right)=x_{5}$, we see that

$$
E_{3}^{*^{\prime},-1} \cong E_{3}^{2 *,-1} \cong H^{*}\left(H^{*}(G ; \mathbb{Z} / 2) ; \mathrm{Sq}^{2}\right) \cong \Lambda\left(v_{4}\right)
$$

where $v_{4}=x_{3} x_{5}$. We easily see $E_{3}^{*, *^{\prime}} \cong E_{\infty}^{*, *^{\prime}}$ for dimensional reasons. Thus the map

$$
q^{*}: W^{*}\left(G_{k}\right) \cong \Lambda\left(z_{3}\right) \rightarrow K O^{2 *}(G) / K U^{2 *}(G) \cong \Lambda\left(v_{4}\right)
$$

is neither injective nor surjective since $\operatorname{deg}\left(v_{4}\right)=4 \operatorname{but} \operatorname{deg}\left(z_{3}\right)=3$.

\section{$\S 5$. Hermitian $K$-theories}

The Balmer-Witt group can be extended to a generalized cohomology theory for the $\mathbb{A}^{1}$-homotopy category as follows. By the work of Hornbostel, Schlichting, Panin and Walter ([Ho], [Sch], [Sch-Tr], [Pa-Wa]), there is an object (spectrum) KO in the stable $\mathbb{A}^{1}$-homotopy category such that the hermitian $K$-theory can be written as the group of morphisms of the stable $\mathbb{A}^{1}$-homotopy category

$$
K O^{*, *^{\prime}}(X) \cong \operatorname{Hom}_{\mathbb{A}^{1}}\left(X, S^{*, *^{\prime}} \wedge K O\right)
$$

where $S^{*, *^{\prime}}$ is the sphere of $\operatorname{deg}=\left(*, *^{\prime}\right)$ in the $\mathbb{A}^{1}$-homotopy category, e.g. $S^{2,1} \cong \mathbb{P}^{1}$. Moreover the Witt group can be written as

$$
W^{i}(X) \cong K O^{i+*, *}(X) \quad \text { for } i-*>0
$$

(that is, $W^{d\left(*, *^{\prime}\right)}(X) \cong K O^{*, *^{\prime}}(X)$ for $\left.w\left(*, *^{\prime}\right)<0\right)$. 
Let $U \rightarrow X \rightarrow T$ be a cofiber sequence in the $\mathbb{A}^{1}$-homotopy category. Then we have the long exact sequence for $W^{*}(-)$ from that for $K O^{*, *^{\prime}}(-)$ when $*>*^{\prime}$ :

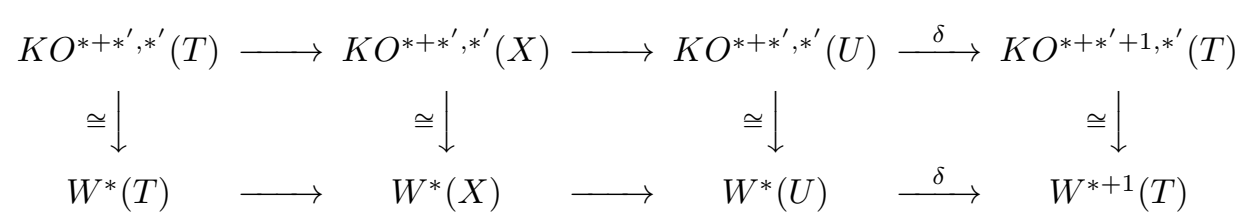

For a given map $X \rightarrow Y$ in the $\mathbb{A}^{1}$-homotopy category, we can define an object $Y / X$ in the $\mathbb{A}^{1}$-homotopy category such that $X \rightarrow Y \rightarrow Y / X$ is a cofiber sequence, and hence we have a long exact sequence

$$
\rightarrow W^{*}(Y / X) \rightarrow W^{*}(Y) \rightarrow W^{*}(X) \stackrel{\delta}{\rightarrow} W^{*+1}(Y / X) \rightarrow \cdots .
$$

Since $K O^{*, *^{\prime}}$-theory has a good product $[\mathrm{Pa}-\mathrm{Wa}]$, and hence $W^{*}(X)$ has a graded ring structure compatible with $K O^{*}(X(\mathbb{C}))$ by the realization map $t_{\mathbb{C}}$ : $K O^{*, *^{\prime}}(X) \rightarrow K O^{*}(X(\mathbb{C}))$ (see [Pa-Wa], or [Vo1, §3.4]), we have a natural map

$$
W^{*}(X) \cong K O^{2 *-1, *-1}(X) \rightarrow K O^{2 *-1}(X(\mathbb{C})) .
$$

Zibrowius [Zi, Theorem 2.5] proved that the above map is an isomorphism when $X$ is cellular and $k=\mathbb{C}$. (Such an isomorphism was first found in [Ya3] when $X$ is the complex Grassmannian.) We give a (slightly different) proof for $k$ algebraically closed.

For the rest of this section, we assume that $k$ is an algebraically closed subfield of $\mathbb{C}$.

Let $X$ be cellular. By definition, $X$ has a filtration by closed subvarieties

$$
\emptyset=Z^{n+1} \subset Z^{n} \subset \cdots \subset Z^{0}=X
$$

such that $Z^{k}-Z^{k+1} \cong \coprod \mathbb{A}^{i_{k}}$. Then we have the cofiber sequence

$$
Z^{i}-Z^{i+1}=\left(Z^{i}-Z^{i+1}\right) /\left(Z^{i}-Z^{i}\right) \stackrel{f}{\rightarrow} X /\left(X-Z^{i}\right) \stackrel{q}{\rightarrow} X /\left(X-Z^{i+1}\right)
$$

and the induced exact sequence

$$
\rightarrow W^{*}\left(X /\left(X-Z^{i+1}\right)\right) \stackrel{q^{*}}{\longrightarrow} W^{*}\left(X /\left(X-Z^{i}\right)\right) \stackrel{f^{*}}{\longrightarrow} W^{*}\left(Z^{i}-Z^{i+1}\right) \stackrel{\delta}{\rightarrow} \cdots .
$$

Since $W^{*}\left(Z^{i}-Z^{i+1}\right) \cong \bigoplus W^{*}(\mathrm{pt})$, by induction we can show that the Zibrowius theorem can be deduced from the following lemma.

Lemma 5.1. Let $k$ be an algebraically closed field. Let $U \rightarrow X \rightarrow T$ be a cofiber sequence in the stable $\mathbb{A}^{1}$-homotopy category. If (1) and (2) below are satisfied for 
both $Y=T, U$, then they also hold for $Y=X$, where

$$
\begin{aligned}
& \text { (1) } K U^{\text {odd }}\left(t_{\mathbb{C}}(Y)\right)=0, \\
& (2) \quad t_{\mathbb{C}}: W^{*}(Y) \cong K O^{2 *}\left(t_{\mathbb{C}}(Y)\right) / K U^{2 *}\left(t_{\mathbb{C}}(Y)\right) \cong K O^{2 *-1}\left(t_{\mathbb{C}}(Y)\right) .
\end{aligned}
$$

Proof. Let us write simply $K O^{*}(-)$ for $K O^{*}\left(t_{\mathbb{C}}(-)\right)$. We consider the following map of long exact sequences:

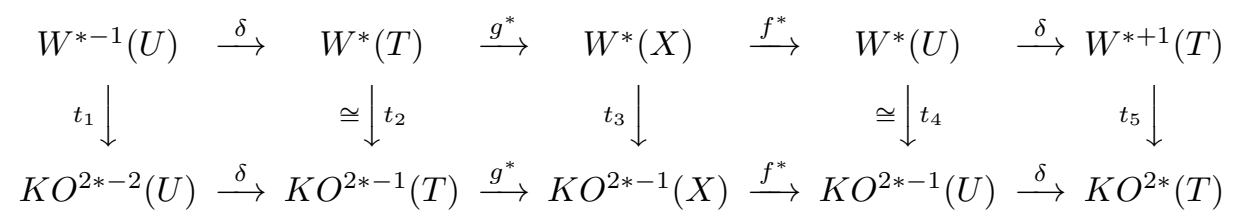

where $t_{i}=t_{\mathbb{C}}: W^{*}(X)=K O^{*+*^{\prime}, *^{\prime}}(Z) \rightarrow K O^{*+*^{\prime}}(Z)$ are the realization maps. That is, $t_{i}: W^{*}(X)=K O^{*+*-1, *-1}(Z) \rightarrow K O^{2 *-1}(Z)$ for $i=2,3,4$ and the map $t_{5}$ is defined from the diagram

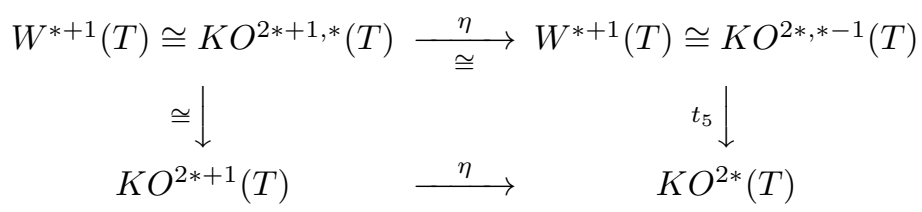

Hence $t_{5}$ is injective. Indeed, $\eta: K O^{\text {odd }}(T) \rightarrow K O^{\text {even }}(T)$ is injective from the Bott exact sequence since $K U^{\text {odd }}(T)=0$ (note that $\eta: K O^{\text {even }}(T) \rightarrow K^{\text {odd }}(T)$ is surjective).

Next we look at the map $t_{1}$. Considering the similar exact sequence for $K U^{*}(-)$ theory, we see $\delta \mid K U^{2 *-2}(U)=0$ from $\delta K U^{2 *-2}(U) \subset K U^{2 *-1}(T)=0$. Hence the map $\delta$ on $K O^{*}$-theories factors as

$$
\delta: K O^{2 *-2}(U) \rightarrow K O^{2 *-2}(U) / K U^{2 *-2}(U) \rightarrow K O^{2 *-1}(T) .
$$

Moreover, we have the diagram

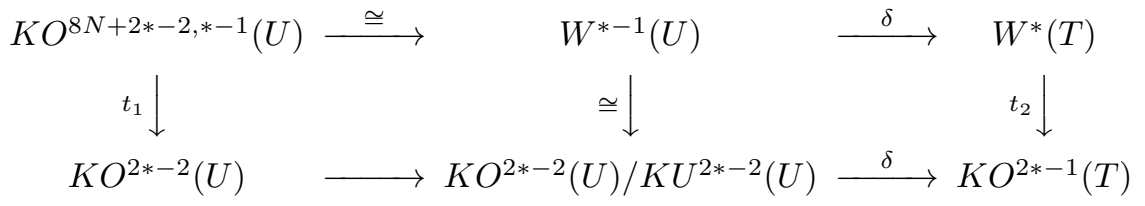

Hence $\delta t_{1}$ surjects to $\operatorname{Ker}\left(g^{*}\right)$. Then we can prove the result from the five lemma and by a diagram chase.

We will study the case which is not cellular but (1), (2) in the above lemma are satisfied. 
Lemma 5.2. Let $k$ be an algebraically closed field in $\mathbb{C}$. Let $U \stackrel{f}{\rightarrow} X \stackrel{g}{\rightarrow} T$ be a cofiber sequence in the stable $\mathbb{A}^{1}$-homotopy category. If (1), (2) in Lemma 5.1 are satisfied for both $Y=X, T$ (resp. $Y=X, U)$, and moreover $g^{*}: K U^{*}\left(t_{\mathbb{C}}(T)\right) \rightarrow$ $K U^{*}\left(t_{\mathbb{C}}(X)\right)$ is injective (resp. $f^{*}: K U^{*}\left(t_{\mathbb{C}}(X)\right) \rightarrow K U^{*}\left(t_{\mathbb{C}}(U)\right)$ is surjective), then (1) and (2) also hold for $Y=U$ (resp. $Y=T)$.

Proof. Consider the map of exact sequences

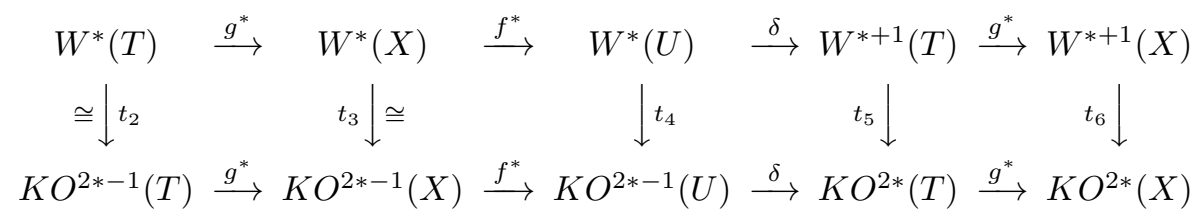

We want to see that $t_{4}$ is an isomorphism. Consider the Bott exact sequence

$$
\begin{aligned}
& 0 \rightarrow W^{*+1}(T) \cong K O^{2 *+1}(T) \stackrel{\eta}{\longrightarrow} K O^{2 *}(T) \longrightarrow K U^{2 *}(T) \\
& g^{*}(1) \downarrow \quad g^{*}(2) \downarrow \quad g^{*}(3) \downarrow \text { injective } \\
& 0 \rightarrow W^{*+1}(X) \cong K O^{2 *+1}(X) \stackrel{\eta}{\longrightarrow} K O^{2 *}(X) \longrightarrow K U^{2 *}(X)
\end{aligned}
$$

where $g^{*}(1), g^{*}(2), g^{*}(3)$ are the maps $g^{*}$ defined on $W^{*}(T), K O^{2 *}(T), K U^{2 *}(T)$ respectively. Here note that $\times \eta \mid K O^{\text {odd }}(-)$ is injective, and $g^{*}(3)$ is also injective by assumption. If $x \in \operatorname{Ker}\left(g^{*}(2)\right)$, then $x \in \operatorname{Im}(\eta)$ from the injectivity of $g^{*}(3)$. Hence we easily see (from the injectivity of $\times \eta$ ) that $\operatorname{Ker}\left(g^{*}(2)\right) \cong \operatorname{Ker}\left(g^{*}(1)\right.$ ), and $t_{4}$ is isomorphic from the five lemma.

The second case is similarly proved by using the following diagram (to see $\left.\operatorname{Im}\left(f^{*}(2)\right) \cong \operatorname{Im}\left(f^{*}(3)\right)\right)$

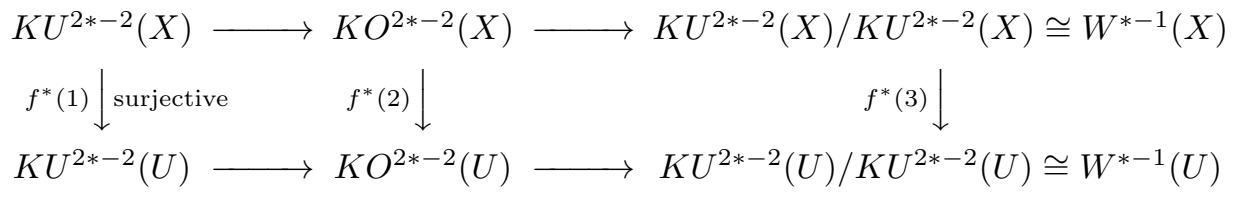

Remark. When $X=G / T^{\ell}, T=\operatorname{Th}\left(t_{\ell}\right)$ and $U=G / T^{\ell-1}$, then of course $t_{\ell}$ : $K U^{*}(T) \rightarrow K U^{*}(X)$ is not injective. In fact, $K U^{*}\left(G / T^{\ell}\right) \cong K U^{*} \otimes H^{*}\left(G / T^{\ell}\right)$ and $g_{\ell}$ (given in Lemma 3.1) is in $\operatorname{Ker}\left(t_{\ell}\right)$. In this case the assumption of the above lemma is not satisfied. Hence $t_{\mathbb{C}} \mid W^{*}(U)$ need not be an isomorphism. (In particular $t_{\mathbb{C}} \mid W^{*}\left(G_{k}\right)$ is not an isomorphism, in general.)

We consider the classifying space $B G$ for a finite group $G$. First consider the case $G=\mathbb{Z} / 2^{r}$. In the stable $\mathbb{A}^{1}$-homotopy category, we have the cofiber sequence 
(see $[$ Vo3, (6.4)] for details)

$$
B \mathbb{Z} / 2^{r} \rightarrow E \stackrel{q_{r}}{\longrightarrow} \operatorname{Th}(E)
$$

where $E=O\left(-2^{r}\right)$ is the $\left(-2^{r}\right)$-th twisted line bundle (of the canonical one) of $\mathbb{P}^{\infty}$, $\operatorname{Th}(E)$ is its Thom space and $q_{r}$ is the composition map with $2^{r}$-fold twist on $\mathbb{P}^{\infty}$ and the quotient map.

Of course $\mathbb{P}^{n}$ is cellular, and (see $[$ Ars] or $[$ Ya1, $\S 7]$ )

$$
W^{*}\left(\mathbb{P}^{n}\right) \cong \begin{cases}\mathbb{Z} / 2\left\{1, y_{n}\right\} & \text { for } \operatorname{deg}\left(y_{n}\right)=n \text { if } n \text { is odd } \\ \mathbb{Z} / 2\{1\}, & \text { otherwise. }\end{cases}
$$

Hence $W^{*}\left(\mathbb{P}^{\infty}\right) \cong \mathbb{Z} / 2$. (For Witt groups of infinite spaces, see the remark after Lemma 6.1 below.) We also see $W^{*}\left(\left(\mathbb{P}^{\infty}\right)^{\times n}\right) \cong \mathbb{Z} / 2$. Moreover $E=O\left(-2^{r}\right)$ represents zero in $\operatorname{Pic}\left(\mathbb{P}^{\infty}\right) / 2$, and so we have the Thom isomorphism

$$
\tilde{W}^{*+1}(\operatorname{Th}(E)) \cong W^{*}(E) \cong W^{*}\left(\mathbb{P}^{\infty}\right) \cong \mathbb{Z} / 2 .
$$

Therefore we have the exact sequence

$$
\rightarrow W^{*-1}\left(\mathbb{P}^{\infty}\right) \stackrel{0}{\rightarrow} W^{*}\left(\mathbb{P}^{\infty}\right) \rightarrow W^{*}\left(B \mathbb{Z} / 2^{r}\right) \rightarrow \cdots
$$

Hence $W^{*}\left(B \mathbb{Z} / 2^{r}\right) \cong \Lambda(x)$ with $\operatorname{deg}(x)=0$. (In fact $x^{2}=0$, since that is the case in the Gille-Pardon spectral sequence $E(\mathrm{GP})_{2}^{*, *^{\prime}}$; see $\S 6$ below.)

For each space $X$, we have the cofiber sequence (see $[\mathrm{Vo} 3, \S 6]$ )

$$
B \mathbb{Z} / 2^{r} \times X \rightarrow E \times X \stackrel{q_{r} \times \mathrm{id}}{\longrightarrow} \mathrm{Th}(E) \times X .
$$

By induction on $i$, starting with $\left(\mathbb{P}^{\infty}\right)^{\times n}$, we can prove

$$
W^{*}\left(\left(\underset{s=1}{\times} B \mathbb{Z} / 2^{r_{s}}\right) \times\left(\mathbb{P}^{\infty}\right)^{\times(n-i)}\right) \cong \Lambda\left(x_{1}, \ldots, x_{i}\right) \quad \text { with } \quad \operatorname{deg}\left(x_{j}\right)=0 .
$$

Theorem 5.3. Let $k$ be algebraically closed. Let $G$ be an abelian 2 -group of rank $n$ i.e. $G \cong \bigoplus_{s=1}^{n} \mathbb{Z} / 2^{r_{s}}$. Then there are isomorphisms

$$
q^{*}: W^{*}(B G) \cong K O^{2 *}(B G) / K U^{2 *}(B G) \cong \Lambda\left(x_{1}, \ldots, x_{n}\right), \quad \operatorname{deg}\left(x_{i}\right)=0 .
$$

Proof. We only need to see the first isomorphism. It is well known that $K U^{*}(B G)$ is torsion free for each compact Lie group $G$. In particular

$$
\times 2^{r_{i}} \mid K U^{*}\left(\left(\underset{s=1}{\mathrm{X}-1} B \mathbb{Z} / 2^{r_{s}}\right) \times\left(\mathbb{P}^{\infty}\right)^{\times(n-i+1)}\right)
$$

is injective. Hence this satisfies the assumptions in Lemma 5.2. Thus (2) in the lemma is satisfied for $U=\left(X_{s=1}^{i} B \mathbb{Z} / 2^{r_{s}}\right) \times\left(\mathbb{P}^{\infty}\right)^{\times(n-i)}$. 
The above isomorphisms were already given in [Ya1] for $G=(\mathbb{Z} / 2)^{\oplus n}$. (However it was not proved that the map $q^{*}$ induces these isomorphisms.)

Let $S$ be a 2-Sylow subgroup of a finite group $G$. It is known that for the inclusion $i: S \hookrightarrow G$, the induced map $g=B i: B S \rightarrow B G$ is a finite covering of order $[G: S]$. Hence the composition map $g_{*} \cdot g^{*}$ is an isomorphism for the Gysin map $g_{*}$, and $W^{*}(B G)$ is a subring of $W^{*}(B S)$.

Corollary 5.4. Let $k$ be algebraically closed. Let $G$ be a finite group having an abelian 2-Sylow subgroup. Then

$$
q^{*}: W^{*}(B G) \cong K O^{2 *}(B G) / K U^{2 *}(B G)
$$

Let $L$ be a line bundle over a smooth scheme $X$ over $k$ and $\operatorname{Th}(L)$ be its Thom class. Here we recall that the Witt group $W^{*}(X ; L)$ can be written by using $\operatorname{Th}(L)$ ([Zi]), namely, $W^{*+1}(X ; L) \cong \tilde{W}^{*}(\operatorname{Th}(L))$.

We will prove the Calmès and Fasel result for an algebraically closed field $k$.

Lemma 5.5 ([Zi, (2.4)]).

$$
H\left(\tilde{H}^{*}(\operatorname{Th}(L) ; \mathbb{Z} / 2) ; \mathrm{Sq}^{2}\right) \cong H\left(H^{*}(X ; \mathbb{Z} / 2) ; \mathrm{Sq}^{2}+c_{1}(L)\right) .
$$

Proof. Recall that the mod (2) cohomology of $\operatorname{Th}(L)$ is

$$
\tilde{H}^{*}(\operatorname{Th}(L) ; \mathbb{Z} / 2) \cong H^{*}(X ; \mathbb{Z} / 2)\left\{c_{1}\right\} \subset H^{*}(X ; \mathbb{Z} / 2)\left\{1, c_{1}\right\} \cong H^{*}(P(L) ; \mathbb{Z} / 2)
$$

where $P(L)$ is the associated projective bundle and $c_{1}$ is the first Chern class so that $c_{1}^{2}=c_{1}(L) c_{1}$. Hence for $x \in H^{*}(X ; \mathbb{Z} / 2)$, we have (by the Cartan formula)

$$
\mathrm{Sq}^{2}\left(x c_{1}\right)=\mathrm{Sq}^{2}(x) c_{1}+x c_{1}(L) c_{1}=\left(\mathrm{Sq}^{2}(x)+c_{1}(L) x\right)\left(c_{1}\right) \text {. }
$$

Each element in $\tilde{H}^{*}(\operatorname{Th}(L) ; \mathbb{Z} / 2)$ can be expressed as $x c_{1}$, and we have the result.

Recall that $\operatorname{gr} H^{*}(G / T ; \mathbb{Z} / 2) \cong P(y) \otimes S(t) /\left(b_{1}, \ldots, b_{\ell}\right)$ and $S(t)=$ $\mathbb{Z} / 2\left[t_{1}, \ldots, t_{\ell}\right]$ as stated in $\S 3$.

Lemma 5.6. For each $0 \neq t \in \mathbb{Z} / 2\left\{t_{1}, \ldots, t_{\ell}\right\}$,

$$
H\left(H^{*}(\mathrm{Th}(t) ; \mathbb{Z} / 2) ; \mathrm{Sq}^{2}\right) \cong H\left(H^{*}(G / T ; \mathbb{Z} / 2) ; \mathrm{Sq}^{2}+t\right)=0 \text {. }
$$

Proof. Let $t=t_{1}$ and $d=\mathrm{Sq}^{2}+t$. Define the degree of $t_{i}$ by $w\left(t_{1}\right)=1$ and $w\left(t_{i}\right)=0$ for $i \geq 2$. Let $D_{i}=\{x \in S(t) \mid w(x) \geq i\}$ and $\operatorname{gr} S(i)=\bigoplus D_{i} / D_{i+1}$. Decompose $\operatorname{gr} S(t)=\mathbb{Z} / 2\left[t_{1}\right] \otimes B$ as a $d$-module with $B=\bigotimes_{i>2} \mathbb{Z} / 2\left[t_{i}\right]$. Then $d=\mathrm{Sq}^{2}+t$ acts on $B$ as $\mathrm{Sq}^{2}+0$ for $i \geq 2$. 
We consider the spectral sequence

$$
E_{2}^{*}=H^{*}(\operatorname{gr} S(t) ; d) \Rightarrow H^{*}(S(t) ; d) .
$$

We easily see that

$$
E_{2}^{*} \cong H^{*}\left(B ; \mathrm{Sq}^{2}\right) \otimes \mathbb{Z} / 2[t] \cong \mathbb{Z} / 2[t]
$$

since $\mathrm{Sq}^{2}: t^{\text {odd }} \mapsto t^{\text {odd }+1}$ and $H^{*}\left(B ; \mathrm{Sq}^{2}\right) \cong \mathbb{Z} / 2$. On the other hand, we see that $\mathrm{Sq}^{2}+t: t^{\text {even }} \mapsto t^{\text {even }+1}$ and $H^{*}(\mathbb{Z} / 2[t] ; d)=0$. Thus we get $H^{*}(S(t) ; d)=0$.

The $\mathrm{Sq}^{2}$-action on $H^{*}(G ; \mathbb{Z} / 2)$ is a derivation $\bmod \left(\mathrm{Sq}^{1}\right)$. Hence $\mathrm{Sq}^{2}$ maps ring generators to ring generators or zero by arguments from differential Hopf algebra (see [Ar1], or see the explanation just before Lemma 6.6 below), i.e. we can take generators $x_{i}$ so that $\mathrm{Sq}^{2}\left(x_{i}\right)=x_{i+1}$ or $\operatorname{Sq}^{2}\left(x_{i}\right)=0$. (Moreover if $\left|x_{i}\right|+2=\left|x_{j}\right|$, then $\mathrm{Sq}^{2}\left(x_{i}\right)=x_{j}$ for simply connected simple Lie groups [Ka].) Let $\operatorname{Sq}^{2}\left(x_{1}\right)=x_{2}$. Write $d_{r}\left(x_{1}\right)=b_{1}$ and $d_{r+2}\left(x_{2}\right)=b_{2} \in S(t)$. Then the Cartan-Serre transgression theorem implies that

$$
\operatorname{Sq}^{2}\left(b_{1}\right)=\operatorname{Sq}^{2}\left(d_{r}\left(x_{1}\right)\right)=d_{r+2}\left(\operatorname{Sq}^{2}\left(x_{1}\right)\right)=d_{r+2}\left(x_{2}\right)=b_{2} \bmod \left(b_{1}\right) .
$$

Similarly if $\mathrm{Sq}^{2}\left(x_{1}\right)=0$, then $\operatorname{Sq}^{2}\left(b_{1}\right)=0 \bmod \left(b_{1}\right)$.

Suppose that $\mathrm{Sq}^{2}\left(b_{1}\right)=0 \bmod \left(b_{1}\right)$ (i.e. $\operatorname{Sq}^{2}\left(x_{1}\right)=0$ by the arguments above). Then $S(t) /\left(b_{1}\right)$ is a $d$-module because

$$
\left(\mathrm{Sq}^{2}+t\right)\left(x b_{1}\right)=\mathrm{Sq}^{2}(x) b_{1}+t x b_{1} \in\left(b_{1}\right)
$$

by the Cartan formula. We consider the short exact sequence

$$
0 \rightarrow S(t) \stackrel{b_{1}}{\longrightarrow} S(t) \rightarrow S(t) /\left(b_{1}\right) \rightarrow 0
$$

of $d$-modules, and consider the induced long exact sequence

$$
\rightarrow H^{*}(S(t) ; d) \rightarrow H^{*}(S(t) ; d) \rightarrow H^{*}\left(S(t) /\left(b_{1}\right) ; d\right) \rightarrow \cdots .
$$

The first and second terms in the above sequence are zero, hence so is the third, that is, $H^{*}\left(S(t) /\left(b_{1}\right) ; d\right)=0$.

Suppose that $\mathrm{Sq}^{2}\left(b_{1}\right) \neq 0$ and take $b_{2}=\operatorname{Sq}^{2}\left(b_{1}\right)$ (i.e. $\left.\operatorname{Sq}^{2}\left(x_{1}\right)=x_{2}\right)$. Then $S(t) /\left(b_{2}\right)$ is a $d$-module and $H^{*}\left(S(t) /\left(b_{2}\right) ; d\right)=0$ by the arguments above. Next consider a short exact sequence of $d$-modules (see also [Ki-Oh])

$$
0 \rightarrow S(t) /\left(b_{2}\right) \stackrel{b_{1}}{\rightarrow} S(t) /\left(b_{2}\right) \rightarrow S(t) /\left(b_{1}, b_{2}\right) \rightarrow 0 .
$$

By considering the induced long exact sequence of $d$-homology, we deduce that $H^{*}\left(S(t) /\left(b_{1}, b_{2}\right) ; d\right)=0$. Similarly, $H^{*}\left(S(t) /\left(b_{1}, \ldots, b_{\ell}\right) ; d\right)=0$. 
For each nonzero element $y$ in $P(y)$, take $\tilde{y} \in H^{*}(G / T ; \mathbb{Z} / 2)$ with $\pi^{*}(\tilde{y})=y$ for the projection $\pi: G \rightarrow G / T$. Define $w(\tilde{y})=0$ and $w\left(t_{j}\right)=1$ for $1 \leq j \leq \ell$. Then the associated graded ring is

$$
\operatorname{gr} H^{*}(G / T ; \mathbb{Z} / 2) \cong P(y) \otimes S(t) /\left(b_{1}, \ldots, b_{\ell}\right)
$$

as stated in $\S 3$. We consider the spectral sequence

$$
E_{2}^{*}=H\left(\operatorname{gr} H^{*}(G / T ; \mathbb{Z} / 2) ; d\right) \Rightarrow H\left(H^{*}(G / T ; \mathbb{Z} / 2) ; d\right) .
$$

By the definition of the filtration, see

$$
E_{2}^{*} \cong H^{*}\left(P(y) ; \mathrm{Sq}^{2}\right) \otimes S(t) /\left(b_{1}, \ldots, b_{\ell}\right)
$$

For each nonzero $y \in H^{*}\left(P(y) ; \mathrm{Sq}^{2}\right)$ and $s \in S(t)$, the differential $d$ acts on $E_{2}^{*}$ as

$$
y \otimes s \mapsto \mathrm{Sq}^{2}(\tilde{y})(1 \otimes s)+y \otimes \mathrm{Sq}^{2}(s)+y \otimes t s=y \otimes d(s),
$$

because, from Lemma 5.7 below, we can take $\tilde{y} \in H^{*}(G / T ; \mathbb{Z} / 2)$ with $\operatorname{Sq}^{2}(\tilde{y})=$ $0 \bmod \left(t_{i} t_{j}\right)$. The fact that $H^{*}\left(S(t) /\left(b_{1}, \ldots, b_{\ell}\right) ; d\right)=0$ implies

$$
H\left(H^{*}(G / T ; \mathbb{Z} / 2) ; d\right)=0
$$

Lemma 5.7. Given an element $y \in \operatorname{Ker}\left(\mathrm{Sq}^{2}\right) \mid P(y) \subset H^{*}(G ; \mathbb{Z} / 2)$, we can take $\tilde{y} \in$ $H^{*}(G / T ; \mathbb{Z} / 2)$ with $\pi^{*}(\tilde{y})=y$ for $\pi: G \rightarrow G / T$ such that $\operatorname{Sq}^{2}(\tilde{y})=0 \bmod \left(t_{i} t_{j}\right)$.

Proof. We have the multiplicative map $\mu: G \times G / T \rightarrow G / T$ defined by $\mu\left(g, g^{\prime} T\right)=$ $g g^{\prime} T$. This map induces the map (see [Tod] for details)

$$
\mu^{*}: H^{*}(G / T ; \mathbb{Z} / 2) \rightarrow H^{*}(G ; \mathbb{Z} / 2) \otimes H^{*}(G / T ; \mathbb{Z} / 2) .
$$

Given $y \in P(y)$, we can take $\tilde{y} \in H^{*}(G / T ; \mathbb{Z} / 2)$ with $\pi^{*}(\tilde{y})=y$ such that

$$
\mu^{*}(\tilde{y})=y \otimes 1+1 \otimes \tilde{y} \bmod \left(t_{i} t_{j}\right)
$$

(adding $t_{k} \tilde{y}^{\prime}$ to $\tilde{y}$ if necessary). So we have

$$
\mu^{*}\left(\operatorname{Sq}^{2}(\tilde{y})\right)=\operatorname{Sq}^{2}(y) \otimes 1+1 \otimes \operatorname{Sq}^{2}(\tilde{y}) \bmod \left(t_{i} t_{j}\right)
$$

We show that if $\operatorname{Sq}^{2}(y)=0$, then $\operatorname{Sq}^{2}(\tilde{y})=0 \bmod \left(t_{i} t_{j}\right)$. Otherwise, $\operatorname{Sq}^{2}(\tilde{y})=$ $y^{\prime} t+\cdots$ for $0 \neq y^{\prime} \in H^{*}(G ; \mathbb{Z} / 2)$ and hence

$$
\mu^{*}\left(\operatorname{Sq}^{2}(\tilde{y})\right)=y^{\prime} \otimes t+\cdots \neq 1 \otimes \operatorname{Sq}^{2}(\tilde{y}) \bmod \left(t_{i} t_{j}\right)
$$

which is a contradiction. 
Theorem 5.8 (Calmès and Fasel theorem for an algebraically closed subfield in $\mathbb{C}$ ). Let $k$ be an algebraically closed field in $\mathbb{C}$ and $G$ be a compact simply connected Lie group. If $0 \neq L \in \operatorname{Pic}\left(G_{k} / T_{k}\right) / 2$, then $W^{*}\left(G_{k} / T_{k} ; L\right)=0$.

Proof. We have

$$
W^{*-1}\left(G_{k} / T_{k} ; L\right) \cong \tilde{W}^{*}(\operatorname{Th}(L)) \cong \tilde{K} O^{2 *}(\operatorname{Th}(L)) / \tilde{K} O^{2 *}(\operatorname{Th}(L)) .
$$

The last term is isomorphic to a subquotient of $H\left(\tilde{H}^{*}(\mathrm{Th}(L) ; \mathbb{Z} / 2) ; \mathrm{Sq}^{2}\right)$, which is isomorphic to $H\left(H^{*}(G / T ; \mathbb{Z} / 2) ; \mathrm{Sq}^{2}+c_{1}(L)\right)=0$ from Lemma 5.6.

For each algebraic group $G$, by Voevodsky [Vo3], we can take smooth schemes $X_{n}$ such that

$$
\operatorname{colim}_{n} X_{n}=B G \quad \text { and } \quad H^{*, *^{\prime}}\left(X_{n} ; \mathbb{Z} / 2\right) \cong H^{*, *^{\prime}}(B G ; \mathbb{Z} / 2) \quad \text { for } *^{\prime} \leq n .
$$

So for $L \in H^{2,1}(B G ; \mathbb{Z} / 2) \cong H^{2,1}\left(X_{n} ; \mathbb{Z} / 2\right)$, we can define

$$
W^{*}(B G ; L)=\lim _{n} W^{*}\left(X_{n} ; L\right) .
$$

Recall gr $H^{*}\left(B \mathbb{Z} / 2^{r}\right) \cong \mathbb{Z} / 2[y] \otimes \Lambda(x)$, and $H^{2,1}\left(B \mathbb{Z} / 2^{r} ; \mathbb{Z} / 2\right) \cong \mathbb{Z} / 2\{y\}$. We easily see that $H\left(\mathbb{Z} / 2[y] \otimes \Lambda(x) ; \mathrm{Sq}^{2}+y\right)=0$ as in the proof of Lemma 5.6. The analogue of the theorem by Calmès and Fasel also holds for abelian 2-groups.

Corollary 5.9. Let $k$ be algebraically closed and let $G \cong \bigoplus_{s=1}^{n} \mathbb{Z} /\left(2^{r_{s}}\right)$. Then

$$
W^{*}(B G ; L)=0 \quad \text { for } 0 \neq L \in H^{2,1}(B G ; \mathbb{Z} / 2) .
$$

\section{$\S 6$. Gille-Pardon spectral sequence}

Balmer and Walter [Ba-Wa, (1)] define the Gersten-Witt complex

$$
0 \rightarrow W(k(X)) \rightarrow \bigoplus_{x \in X^{(1)}} W(k(x)) \rightarrow \cdots \rightarrow \bigoplus_{x \in X^{(n)}} W(k(x)) \rightarrow 0
$$

where $X^{(i)}$ is the set of closed points of codimension $i$ in $X, k(X)$ is the function field of $X$ and $k(x)$ is the residue field for the closed point $x$. Let $H^{*}(W(X))$ denote the cohomology group of the above cochain complex, with $W(k(X))$ in degree 0 . Then Balmer-Walter constructed the spectral sequence [Ba-Wa, Theorem]

$$
E(\mathrm{BW})_{2}^{*, *^{\prime}} \cong\left\{\begin{array}{l}
H^{*}(W(X))\left(*^{\prime}=0 \bmod (4)\right) \\
0 \quad\left(*^{\prime} \neq 0 \bmod (4)\right)
\end{array} \quad \Rightarrow W^{*+*^{\prime}}(X) .\right.
$$

By the affirmative answer to the Milnor conjecture of quadratic forms by Orlov-Vishik-Voevodsky [Or-Vi-Vo, Theorem 4.1], we have an isomorphism of 
graded rings $H_{\mathrm{et}}^{*}(k(x) ; \mathbb{Z} / 2) \cong \operatorname{gr} W(k(x))$. Using this fact, Pardon and Gille constructed ([Pa, Corollary 0.13] and [Gi]) a spectral sequence

$$
E(\mathrm{GP})_{2}^{*, *^{\prime}} \cong H_{\mathrm{Zar}}^{*}\left(X ; H_{\mathbb{Z} / 2}^{*^{\prime}}\right) \Rightarrow H^{*}(W(X)) \cong E(\mathrm{BW})_{2}^{*, 4 t}
$$

so that the differential $d_{r}$ has degree $(1, r-1)$ for $r \geq 2$. Here $H_{\mathbb{Z} / 2}^{*^{\prime}}$ is the Zariski sheaf associated to the presheaf $V \mapsto H_{\mathrm{et}}^{*^{\prime}}(V ; \mathbb{Z} / 2)$ for any open subscheme $V$ of $X$.

The above sheaf cohomology $H_{\mathrm{Zar}}^{*}\left(X ; H_{\mathbb{Z} / 2}^{*^{\prime}}\right)$ is related to the motivic cohomology $H^{*, *^{\prime}}(X ; \mathbb{Z} / 2)=\bigoplus_{r, s \in \mathbb{Z}} H^{r, s}(X ; \mathbb{Z} / 2)$ (for details, see [Vo1]-[Vo3]) as follows. Recall that $\tau \in H^{0,1}(\operatorname{Spec}(k) ; \mathbb{Z} / 2) \cong \mathbb{Z} / 2$ be a generator. (For example, if $k$ is algebraically closed, then $H^{*, *^{\prime}}(\operatorname{Spec}(k) ; \mathbb{Z} / 2) \cong \mathbb{Z} / 2[\tau]$.) Then we get the long exact sequence from the solution of the Beilinson-Lichtenbaum conjecture ([To, Theorem 1.3], [Or-Vi-Vo, Lemma 2.4(5)]),

$$
\begin{aligned}
\rightarrow H^{*, *^{\prime}-1}(X ; \mathbb{Z} / 2) \stackrel{\times \tau}{\longrightarrow} & H^{*, *^{\prime}}(X ; \mathbb{Z} / 2) \\
& \rightarrow H_{\mathrm{Zar}}^{*-*^{\prime}}\left(X ; H_{\mathbb{Z} / 2}^{*^{\prime}}\right) \rightarrow H^{*+1, *^{\prime}-1}(X ; \mathbb{Z} / 2) \stackrel{\times \tau}{\longrightarrow} \cdots .
\end{aligned}
$$

All elements in the above cohomology groups are 2-torsion, and so we have the following additive isomorphisms:

\section{Lemma 6.1.}

$$
\begin{aligned}
E(\mathrm{GP})_{2}^{*-*^{\prime}, *^{\prime}} & \cong H_{\mathrm{Zar}}^{*-*^{\prime}}\left(X ; H_{\mathbb{Z} / 2}^{*^{\prime}}\right) \\
& \cong H^{*, *^{\prime}}(X ; \mathbb{Z} / 2) /\left.(\operatorname{Im}(\tau)) \oplus \operatorname{Ker}(\tau)\right|_{H^{*+1, *^{\prime}-1}(X ; \mathbb{Z} / 2)} .
\end{aligned}
$$

Remark. Let $X_{n}$ be smooth and $\operatorname{colim}_{n} X_{n}=X$. Suppose that $\bigoplus_{s} H^{*}\left(X_{n} ; H_{\mathbb{Z} / 2}^{s}\right)$ is a finite group for each $*$. (Note $H_{\mathrm{Zar}}^{*}\left(X_{n} ; H_{\mathbb{Z} / 2}^{*^{\prime}}\right)=0$ for $*>\operatorname{dim}\left(X_{n}\right)$.) Then $\bigoplus_{m, s} E(\mathrm{GP})_{r}^{m, s}\left(X_{n}\right)$ is a finite group, and so is $\bigoplus_{m} E(\mathrm{BW})_{\infty}^{m, 0}$. Therefore $W^{*}\left(X_{n}\right)$ is a finite group for each $* \in \mathbb{Z} / 4$. So $\lim ^{1}=0$, and we have

$$
W^{*}(X) \cong \lim _{n} W^{*}\left(X_{n}\right) .
$$

It is well known that $\mathrm{CH}^{*}(X) \cong H^{2 *, *}(X ; \mathbb{Z})$ [Vo2, Corollary 2]). Recall that $H^{*, *^{\prime}}(X ; \mathbb{Z} / 2)=0$ when $*>2 *^{\prime}$ (i.e. when the weight degree is $<0$ [Vo1, Corollary 2.3]). Since $H^{2 *+1, *}(X ; \mathbb{Z})=0$, from the Bockstein exact sequence we have $H^{2 *, *}(X ; \mathbb{Z} / 2) \cong H^{2 *, *}(X ; \mathbb{Z}) / 2$. In particular,

$$
\begin{aligned}
E(\mathrm{GP})_{2}^{* * *} & \cong H_{\mathrm{Zar}}^{*}\left(X ; H_{\mathbb{Z} / 2}^{*}\right) \\
& \cong H^{2 *, *}(X ; \mathbb{Z} / 2) /\left.\left(\tau \cdot H^{2 *, *-1}(X ; \mathbb{Z} / 2)\right) \oplus \operatorname{Ker}(\tau)\right|_{H^{2 *+1, *-1}(X ; \mathbb{Z} / 2)} .
\end{aligned}
$$

Here $H^{2 *, *-1}(X ; \mathbb{Z} / 2)=H^{2 *+1, *-1}(X ; \mathbb{Z} / 2)=0$ since the weight degree is negative. Hence

$$
E(\mathrm{GP})_{2}^{* * *} \cong H^{2 *, *}(X ; \mathbb{Z} / 2) \cong \mathrm{CH}^{*}(X) / 2 .
$$


Similarly,

$$
E(\mathrm{GP})_{2}^{*, *+1} \cong H_{\mathrm{Zar}}^{*}\left(X ; H_{\mathbb{Z} / 2}^{*+1}\right) \cong H^{2 *, *+1}(X ; \mathbb{Z} / 2)
$$

On $H^{*, *^{\prime}}(X ; \mathbb{Z} / 2)$, we can define the cohomology operation $\mathrm{Sq}^{i}$ from Voevodsky $\left([\mathrm{Vo} 3, \S 9],[\mathrm{Vo} 1, \S 3.3]\right.$ ) (or Brosnan $[\mathrm{Br}]$ for $\mathrm{CH}^{*}(X) / 2$ ). This $\mathrm{Sq}^{i}$ is compatible with that in the usual (topological) $\bmod 2$ cohomology via the realization map $t_{\mathbb{C}}$ when $k \subset \mathbb{C}\left(\left[\right.\right.$ Vo1, §3.4]). Moreover $\mathrm{Sq}^{2} \mathrm{Sq}^{2}=\tau \mathrm{Sq}^{3} \mathrm{Sq}^{1}$ [Vo3, Theorem 10.2]). Then Totaro proved

Lemma 6.2 ([To, Theorem 1.1]). If $x \in E(\mathrm{GP})_{2}^{*, *} \cong \mathrm{CH}^{*}(X) / 2$, then $d_{2}(x)=$ $\mathrm{Sq}^{2}(x)$.

We assume the following throughout this section:

Assumption 6.3. If $x \in E(\mathrm{GP})_{2}^{*, *+1} \cong H^{2 *+1, *+1}(X ; \mathbb{Z} / 2)$, then $d_{2}(x)=\operatorname{Sq}^{2}(x)$.

Since $W^{*-*^{\prime}}(X)=K O^{*, *^{\prime}}(X)$ for $*>2 *^{\prime}$ has the ring structure from the results by Schlichting and Panin-Walter, $W^{*}(-)$ is a multiplicative cohomology theory. So $W^{*}(X)$ is a $W^{*}(\mathrm{pt})$-algebra, i.e. we have the cup product via the maps

$$
W^{*}(X) \otimes W^{*^{\prime}}(X) \rightarrow W^{*+*^{\prime}}(X \times X) \stackrel{\Delta^{*}}{\longrightarrow} W^{*+*^{\prime}}(X)
$$

where $\Delta: X \rightarrow X \times X$ is the diagonal map.

For a map $X \rightarrow Y$ in the $\mathbb{A}^{1}$-homotopy category, we have an object $Y / X$ such that $X \rightarrow Y \rightarrow Y / X$ is a cofiber sequence and we have the long exact sequence

$$
\rightarrow \tilde{W}^{*}(Y / X) \rightarrow W^{*}(Y) \rightarrow W^{*}(X) \stackrel{\delta}{\rightarrow} \tilde{W}^{*+1}(Y / X) \rightarrow \cdots .
$$

The coboundary map $\delta$ is a derivation in the following sense. There is a commutative diagram

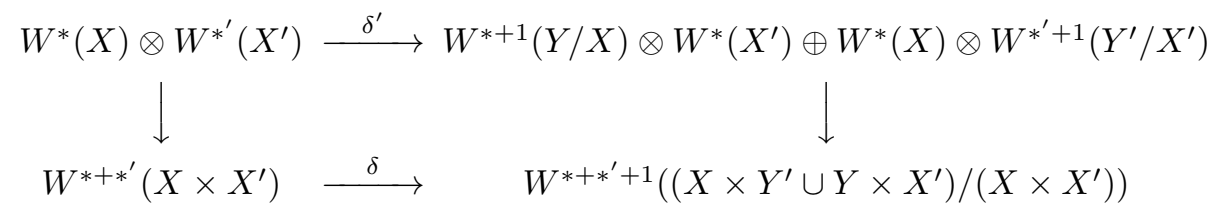

where $\delta^{\prime}(a \otimes b)=\delta(a) \otimes b+a \otimes \delta(b)$ from the standard arguments as in the topological cases [Ar2, (11.11) and (27.4)].

Let us consider a filtration (chain) of closed subvarieties

$$
\mathcal{Z}: Z^{n} \subset Z^{n-1} \subset \cdots \subset Z^{1} \subset X
$$

such that $\operatorname{codim}_{X}\left(Z^{i}\right) \geq i$ and $Z^{i}-Z^{i+1} \subset X-Z^{i+1}$ are regular embeddings. Here we have a cofiber sequence

$$
Z^{i}-Z^{i+1} \stackrel{f}{\hookrightarrow} X /\left(X-Z^{i}\right) \stackrel{g}{\rightarrow} X /\left(X-Z^{i+1}\right)
$$


and a long exact sequence (as stated in $\S 5$ )

$$
\rightarrow W^{*}\left(X /\left(X-Z^{i+1}\right)\right) \stackrel{g^{*}}{\rightarrow} W^{*}\left(X /\left(X-Z^{i}\right)\right) \stackrel{f^{*}}{\rightarrow} W^{*}\left(Z^{i}-Z^{i+1}\right) \stackrel{\delta}{\rightarrow} \cdots .
$$

Then we consider the diagram

$$
\begin{aligned}
& W^{*}(X) \stackrel{g^{*}}{\longleftarrow} W^{*}\left(X /\left(X-Z^{1}\right)\right) \stackrel{g^{*}}{\longleftarrow} W^{*}\left(X /\left(X-Z^{2}\right)\right) \stackrel{g^{*}}{\longleftarrow} \cdots \\
& \searrow f^{*} \quad \uparrow \delta \quad \searrow f^{*} \quad \uparrow \delta \\
& W^{*}\left(X-Z^{1}\right) \stackrel{f^{*} \delta}{\longrightarrow} W^{*}\left(Z^{1}-Z^{2}\right) \stackrel{f^{*} \delta}{\longrightarrow} \cdots
\end{aligned}
$$

The Gersten-Witt complex is constructed by taking the limit of the above diagram (limit of exact couples [Bl-Og], [Co-Ho-Ka], [Ba-Wa], [Pa]) in the following sense. We have the commutative diagram

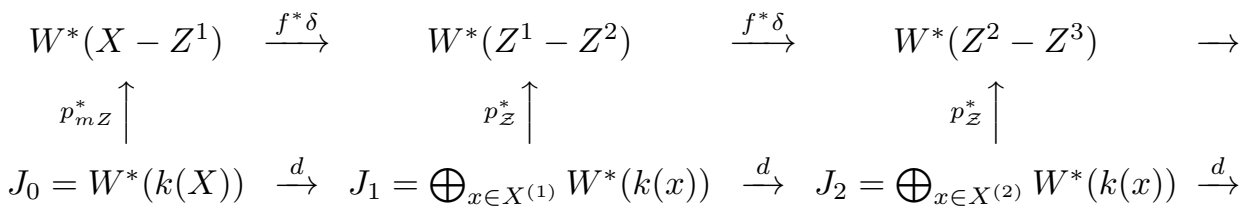

where $X^{(i)}$ is the set of closed points of codimension $i$ in $X, k(X)$ is the function field of $X$ and $k(x)$ is the residue field for the closed point $x$. Moreover we have a natural map

$$
\bigoplus_{x \in X^{(i)}, x^{\prime} \in X^{\prime(j)}} W^{*}(k(x)) \otimes W^{*^{\prime}}\left(k\left(x^{\prime}\right)\right) \rightarrow \bigoplus_{x \in\left(X \times X^{\prime}\right)^{(i+j)}} W^{*+*^{\prime}}(k(x)) .
$$

Then we have the isomorphism

$$
J_{i} \cong \lim _{\mathcal{Z}} W^{*}\left(Z_{i}-Z_{i+1}\right) \subset \Pi_{\mathcal{Z}} W^{*}\left(Z_{i}-Z_{i+1}\right) .
$$

where $\mathcal{Z}$ runs over all chains in $X$ as above. Hence each element $a \in J_{i}$ can be written as $\Pi_{\mathcal{Z}}\left(a_{\mathcal{Z}}\right) \in \Pi_{\mathcal{Z}} W^{*}\left(Z_{i}-Z_{i+1}\right)$ with $a_{\mathcal{Z}}=p_{\mathcal{Z}}^{*}(a)$. The differential is given by $d(a)=\Pi_{\mathcal{Z}}\left(f^{*} \delta\left(a_{\mathcal{Z}}\right)\right)$. Therefore we obtain the following lemma by using that the coboundary map $\delta$ is a derivation in the sense above.

Lemma 6.4. The differential d of the Gersten-Witt complex is a derivation.

The Gille-Pardon spectral sequence is constructed from the associated graded ring giving filtration $I^{s} W^{*}(k(x))$ by the fundamental ideal $I$ (which is the kernel of the rank homomorphism rk $\left.W^{0}(k(x)) \rightarrow \mathbb{Z} / 2\right)$ and using the Milnor conjecture (solved in $[\mathrm{Or}-\mathrm{Vi}-\mathrm{Vo}])$ gr $W^{0}(k(x)) \cong H_{\mathrm{et}}^{*}(k(x) ; \mathbb{Z} / 2)$. Hence the differential of the Gille-Pardon spectral sequence is deduced from the differential $d$ of the GerstenWitt complex. Hence all differentials of the Gille-Pardon spectral sequence are derivations. 
Next consider the Balmer-Walter spectral sequence. Its differential is defined (by the standard arguments for constructing a spectral sequence from an exact couple)

$$
d_{r}(a)=\Pi_{\mathcal{Z}}\left(d_{r}\left(a_{\mathcal{Z}}\right)\right)=\Pi_{\mathcal{Z}}\left(f^{*}\left(g^{*}\right)^{-r+1} \delta\left(a_{\mathcal{Z}}\right)\right)
$$

which is a derivation since $\delta$ is a derivation and $f^{*}, g^{*}$ are ring homomorphisms. Hence all differentials of the Balmer-Walter spectral sequence are derivations. Thus we have the following lemma.

Lemma 6.5. The spectral sequences $E(\mathrm{GP})_{r}^{*, *^{\prime}}$ and $E(\mathrm{BW})_{r}^{*, *^{\prime}}$ are those of graded rings, i.e. all differentials are derivations.

For the rest of this paper, we assume that $k$ is an algebraically closed field in $\mathbb{C}\left(\right.$ i.e. $\left.H^{*, *^{\prime}}(\mathrm{pt}) \cong \mathbb{Z} / 2[\tau]\right)$.

For $X=G_{k}, Y=G_{k}^{\prime}$, from Corollary 3.6 we have

$$
H^{*, *^{\prime}}(X \times Y ; \mathbb{Z} / 2) \cong H^{*, *^{\prime}}(X ; \mathbb{Z} / 2) \otimes_{\mathbb{Z} / 2[\tau]} H^{*, *^{\prime}}(Y ; \mathbb{Z} / 2) .
$$

Thus from Lemma 6.1, we have

$$
\begin{aligned}
& E(\mathrm{GP})_{2}^{*-*^{\prime}, *^{\prime}}(X \times Y) \cong H_{\mathrm{Zar}}^{*-*^{\prime}}\left(X \times Y ; H_{\mathbb{Z} / 2}^{*^{\prime}}\right) \\
& \quad \cong H^{*, *^{\prime}}(X \times Y ; \mathbb{Z} / 2) /(\tau) \cong H^{*, *^{\prime}}(X ; \mathbb{Z} / 2) /(\tau) \otimes H^{*, *^{\prime}}(Y ; \mathbb{Z} / 2) /(\tau) \\
& \quad \cong E(\mathrm{GP})_{2}^{*-*^{\prime}, *^{\prime}}(X) \otimes E(\mathrm{GP})_{2}^{*-*^{\prime}, *^{\prime}}(Y) .
\end{aligned}
$$

By the definition of the spectral sequence and the Künneth formula (see [Ar1] or $[$ Tod] $)$,

$$
\begin{aligned}
E(\mathrm{GP})_{3}^{*, *^{\prime}}(X \times Y) & =H\left(E(\mathrm{GP})_{2}^{*, *^{\prime}}(X \times Y) ; d_{2}\right) \\
& \cong H\left(E(\mathrm{GP})_{2}^{*, *^{\prime}}(X) \otimes E(\mathrm{GP})_{2}^{*, *^{\prime}}(Y) ; d_{2}\right) \\
& \cong H\left(E(\mathrm{GP})_{2}^{*, *^{\prime}}(X) ; d_{2}\right) \otimes H\left(E(\mathrm{GP})_{2}^{*, *^{\prime}}(Y) ; d_{2}\right) \\
& \cong E(\mathrm{GP})_{3}^{*, *^{\prime}}(X) \otimes E(\mathrm{GP})_{3}^{*, *^{\prime}}(Y)
\end{aligned}
$$

By using the Künneth formula, each page of the spectral sequences has the same property for all $r \geq 2$ :

$$
\begin{aligned}
& E(\mathrm{GP})_{r}^{*, *^{\prime}}(X \times Y) \cong E(\mathrm{GP})_{r}^{*, *^{\prime}}(X) \otimes E(\mathrm{GP})_{r}^{*, *^{\prime}}(Y), \\
& E(\mathrm{BW})_{r}^{*, *^{\prime}}(X \times Y) \cong E(\mathrm{BW})_{r}^{*, *^{\prime}}(X) \otimes E(\mathrm{BW})_{r}^{*, *^{\prime}}(Y) .
\end{aligned}
$$

Therefore $\operatorname{gr} W^{*}(X \times Y) \cong \operatorname{gr} W^{*}(X) \otimes \operatorname{gr} W^{*}(Y)$. Hence the ring map $W^{*}(X) \otimes$ $W^{*}(Y) \rightarrow W^{*}(X \times Y)$ is bijective, and it induces an isomorphism

$$
W^{*}(X) \otimes W^{*}(Y) \cong W^{*}(X \times Y) .
$$


When $X=G_{k}$, the product $\mu: G_{k} \times G_{k} \rightarrow G_{k}$ induces a coproduct map

$$
\mu^{*}: W^{*}\left(G_{k}\right) \rightarrow W^{*}\left(G_{k} \times G_{k}\right) \cong W^{*}\left(G_{k}\right) \otimes W^{*}\left(G_{k}\right) .
$$

So $W^{*}\left(G_{k}\right)$ is a Hopf algebra. Moreover $H_{\mathrm{Zar}}^{*}\left(G_{k} ; H_{\mathbb{Z} / 2}^{*^{\prime}}\right)$ is also a Hopf algebra with the coproduct similarly defined by $\mu$. Then each page of the spectral sequences is an (induced) Hopf algebra (by the Künneth formula above). Indeed we have the coproduct

$$
\mu^{*, *^{\prime}}: E_{r}^{*, *^{\prime}}\left(G_{k}\right) \rightarrow E_{r}^{*, *^{\prime}}\left(G_{k}\right) \otimes E_{r}^{*, *^{\prime}}\left(G_{k}\right) .
$$

In particular, $E(\mathrm{BW})_{\infty}^{*, *^{\prime}}$ is isomorphic to $\operatorname{gr} W^{*}\left(G_{k}\right)$ as Hopf algebras. Thus the spectral sequences for $G_{k}$ are differential Hopf algebras. It is known (e.g. Araki [Ar1], Toda [Tod]) that in a differential Hopf algebra, the image of the differential of a ring generator (indecomposable element) is also a ring generator or zero. We can identify gr $E_{r}^{*, *^{\prime}}$ to be primitively generated (namely, we can take generators to be primitive in $g r E_{r}^{*, *^{\prime}}$ ), and use the fact that the image of the differential of a primitive element is primitive.

Lemma 6.6. Let $k$ be an algebraically closed subfield of $\mathbb{C}$. Then the Witt ring $W^{*}\left(G_{k}\right)$ is a Hopf algebra. So the spectral sequences $E(\mathrm{GP})_{r}^{*, *^{\prime}}$ and $E(\mathrm{BW})_{r}^{*, *^{\prime}}$ are differential Hopf algebras, which implies that the differential $d_{r}$ sends ring generators to ring generators or zero.

From Corollary 3.4, we have

$$
H^{*, *^{\prime}}\left(G_{k} ; \mathbb{Z} / 2\right) \cong \mathbb{Z} / 2[\tau] \otimes P(y) \otimes \Delta\left(x_{1}, \ldots, x_{\ell}\right)
$$

with $w(P(y))=0$ and $w\left(x_{i}\right)=1$. (Hence $x_{i}^{2} \in \tau^{j} P(y)$ for some $j \geq 1$.) So we get

$$
\begin{gathered}
H^{*, *^{\prime}}\left(G_{k} ; \mathbb{Z} / 2\right) /(\operatorname{Im}(\tau)) \cong P(y) \otimes \Lambda\left(x_{1}, \ldots, x_{\ell}\right), \\
\left.\operatorname{Ker}(\tau)\right|_{H^{*, *^{\prime}}\left(G_{k} ; \mathbb{Z} / 2\right)}=0 .
\end{gathered}
$$

Thus we get a bigraded ring isomorphism

$$
E(\mathrm{GP})_{2}^{*, *^{\prime}} \cong P(y) \otimes \Lambda\left(x_{1}, \ldots, x_{\ell}\right)
$$

with $\operatorname{deg}(z)=\left(\frac{1}{2}|z|, \frac{1}{2}|z|\right)$ for $z \in P(y)$ and $\operatorname{deg}\left(x_{i}\right)=\left(\frac{1}{2}\left(\left|x_{i}\right|-1\right), \frac{1}{2}\left(\left|x_{i}\right|+1\right)\right)$.

On the other hand, we give a (coniveau) filtration of $H^{*}(G ; \mathbb{Z} / 2)$ by $F_{i}=\{x \mid$ $w(x) \leq i\}$ and denote by gr $H^{*}(G ; \mathbb{Z} / 2)=\bigoplus F_{i} / F_{i-1}$ the associated algebra. Then we have a ring isomorphism

$$
\operatorname{gr} H^{*}(G ; \mathbb{Z} / 2) \cong P(y) \otimes \Lambda\left(x_{1}, \ldots, x_{\ell}\right) .
$$

Since $\mathrm{Sq}^{2} \mathrm{Sq}^{2}=\mathrm{Sq}^{3} \mathrm{Sq}^{1}=0, \mathrm{Sq}^{2}$ defines a differential of $\operatorname{gr} H^{*}(G ; \mathbb{Z} / 2)$. Let us write its homology as $H^{*}\left(G ; \mathrm{Sq}^{2}\right)$ with $\operatorname{deg}\left(y_{i}\right)=\frac{1}{2}\left|y_{i}\right|$ and $\operatorname{deg}\left(x_{i}\right)=\frac{1}{2}\left(\left|x_{i}\right|-1\right)$. 
Then we have an isomorphism of differential Hopf algebras

$$
E(\mathrm{GP})_{2}^{*, *^{\prime}} \cong \operatorname{gr} H^{*}(G ; \mathbb{Z} / 2)
$$

with $w\left(y_{i}\right)=0$ and $w\left(x_{i}\right)=1$. From the result of Totaro and Assumption 6.3, we have

$$
E(\mathrm{GP})_{3}^{*, *^{\prime}} \cong H\left(\operatorname{gr} H^{*^{\prime \prime}}(G ; \mathbb{Z} / 2) ; \mathrm{Sq}^{2}\right)=H^{*}\left(G ; \mathrm{Sq}^{2}\right) .
$$

Recall that the degree of the differential is given by $\operatorname{deg}\left(d_{r}\right)=(1, r-1)$. For $x \in E(\mathrm{GP})_{r}^{*, *^{\prime}}$, let us write its first degree $*$ as f.deg $(x)$. Here we consider the following assumption (which is proved in $\S 7$ below).

Assumption 6.7. There is an algebra isomorphism (for G simply connected)

$$
\begin{gathered}
E(\mathrm{GP})_{3}^{*, *^{\prime}} \cong H^{*}\left(G ; \mathrm{Sq}^{2}\right) \cong \Lambda\left(z_{1}, \ldots, z_{s}\right) \text { with } \mathrm{f.deg}\left(z_{i}\right) \text { odd } \\
\text { or } E(\mathrm{GP})_{4}^{*, *^{\prime}} \cong H^{*}\left(H^{*}\left(G ; \mathrm{Sq}^{2}\right) ; d_{3}\right) \cong \Lambda\left(z_{1}, \ldots, z_{r}\right)
\end{gathered}
$$

For $G=S O(n)$, there is an algebra isomorphism

$$
E(\mathrm{GP})_{3}^{*, *^{\prime}} \cong H^{*}\left(G ; \mathrm{Sq}^{2}\right) \cong \Lambda\left(x_{0}, z_{1}, \ldots, z_{t}\right), \quad \text { f.deg }\left(x_{0}\right)=0 .
$$

Lemma 6.8. Let $G$ be a compact simply connected Lie group. Then there is a spectral sequence

$$
E_{2} \cong H^{*}\left(G ; \mathrm{Sq}^{2}\right) \Rightarrow H^{*}\left(G / T ; \mathrm{Sq}^{2}\right)
$$

Proof. (Recall the proof of Lemma 5.6 and see also [Ki-Oh, §2].) Let us write $d=\mathrm{Sq}^{2}$ for simplicity. Recall $S(t) \cong \bigotimes_{i=1}^{\ell} \mathbb{Z} / 2\left[t_{i}\right]$. We set $\operatorname{deg}\left(t_{i}\right)=1$ here to be compatible with the degree of $W^{*}(-)$. We get

$$
H^{*}(S(t) ; d) \cong \bigotimes_{i=1}^{\ell} H^{*}\left(\mathbb{Z} / 2\left[t_{i}\right] ; d\right) \cong \mathbb{Z} / 2
$$

since $H^{*}\left(\mathbb{Z} / 2[t] ; \mathrm{Sq}^{2}\right) \cong \mathbb{Z} / 2$, for $\mathrm{Sq}^{2}: t^{\text {odd }} \mapsto t^{\text {odd }+1}$.

Suppose that $\operatorname{Sq}^{2}\left(b_{1}\right)=0 \bmod \left(b_{1}\right)$ (i.e. $\operatorname{Sq}^{2}\left(x_{1}\right)=0$ ). Then $S(t) /\left(b_{1}\right)$ is a $d$-module. We consider the short exact sequence

$$
0 \rightarrow S(t) \stackrel{b_{1}}{\longrightarrow} S(t) \rightarrow S(t) /\left(b_{1}\right) \rightarrow 0
$$

of $d$-modules, and consider the induced long exact sequence

$$
\rightarrow H^{*}(S(t) ; d) \stackrel{\times b_{1}}{\longrightarrow} H^{*}(S(t) ; d) \rightarrow H^{*}\left(S(t) /\left(b_{1}\right) ; d\right) \rightarrow \cdots .
$$

The first and second terms in the above sequence are isomorphic to $\mathbb{Z} / 2$ and $\times b_{1}=0$. Hence

$$
H^{*}\left(S(t) /\left(b_{1}\right) ; d\right) \cong \mathbb{Z} / 2\left\{1, \hat{b}_{1}\right\} \cong \Lambda\left(\hat{b}_{1}\right)
$$


Here $d\left(\hat{b}_{1}\right)=b_{1}$ and $\hat{b}_{1}$ is a cycle in $S(t) /\left(b_{1}\right)$ (but not in $S(t)$ ), and $\hat{b}_{1}^{2} \in \operatorname{Im}(d)$ $\subset S(t)$.

Suppose that $\mathrm{Sq}^{2}\left(b_{1}\right) \neq 0$. Then we can take $b_{2}=\mathrm{Sq}^{2}\left(b_{1}\right)$ (i.e. $\left.\mathrm{Sq}^{2}\left(x_{1}\right)=x_{2}\right)$. Then $S(t) /\left(b_{2}\right)$ is a $d$-module and $H^{*}\left(S(t) /\left(b_{2}\right) ; d\right) \cong \Lambda\left(\hat{b}_{2}\right)$; here we can take $\hat{b}_{2}=b_{1}$, by the arguments above. Recall $b_{1}, b_{2}$ is a regular sequence in $S(t)$. We consider a short exact sequence

$$
0 \rightarrow S(t) /\left(b_{2}\right) \stackrel{b_{1}}{\longrightarrow} S(t) /\left(b_{2}\right) \rightarrow S(t) /\left(b_{1}, b_{2}\right) \rightarrow 0,
$$

and the induced long exact sequence of $d$-homology,

$$
\rightarrow \Lambda\left(b_{1}\right) \stackrel{b_{1}}{\longrightarrow} \Lambda\left(b_{1}\right) \rightarrow H^{*}\left(S(t) /\left(b_{1}, b_{2}\right) ; d\right) \rightarrow \cdots .
$$

Hence

$$
H^{*}\left(S(t) /\left(b_{1}, b_{2}\right) ; d\right) \cong \Lambda\left(\hat{b}_{1,2}\right), \quad d\left(\hat{b}_{1,2}\right)=b_{1}^{2} .
$$

Similarly, we can compute $H^{*}\left(S(t) /\left(b_{1}, \ldots, b_{\ell}\right) ; d\right) \cong \Lambda\left(\bar{b}_{1^{\prime}}, \ldots, \bar{b}_{k^{\prime}}\right)$. Here $\bar{b}_{i}$ is defined by $\bar{b}_{i}=\hat{b}_{i}$ if $x_{i} \notin \operatorname{Im}\left(\mathrm{Sq}^{2}\right)$, and $\bar{b}_{i}=\hat{b}_{i-1, i}$ if $\mathrm{Sq}^{2}\left(x_{i-1}\right)=x_{i}$.

On the other hand, we consider the homology of $\Lambda\left(x_{1}, \ldots, x_{\ell}\right)$. If $\operatorname{Sq}^{2}\left(x_{1}\right)=0$, then $H^{*}\left(\Lambda\left(x_{1}\right) ; d_{1}\right) \cong \Lambda\left(x_{1}\right)$. If $\mathrm{Sq}^{2}\left(x_{1}\right)=x_{2}$, then

$$
H^{*}\left(\Lambda\left(x_{1}, x_{2}\right), d\right) \cong \Lambda\left(x_{1,2}\right) \quad \text { with } \quad x_{1,2}=x_{1} x_{2} .
$$

Similarly we have an isomorphism $H^{*}\left(\Lambda\left(x_{1}, \ldots, x_{\ell}\right) ; d\right) \cong \Lambda\left(\bar{x}_{1^{\prime}}, \ldots, \bar{x}_{k^{\prime}}\right)$. Here $\bar{x}_{i}$ is defined by $\bar{x}_{i}=x_{i}$ if $x_{i} \notin \operatorname{Im}\left(\mathrm{Sq}^{2}\right)$, and $\bar{x}_{i}=x_{i-1} x_{i}$ if $\mathrm{Sq}^{2}\left(x_{i-1}\right)=x_{i}$.

Thus we can construct an isomorphism (mapping $\bar{b}_{i} \mapsto \bar{x}_{i}$ )

$$
H^{*}\left(\Lambda\left(x_{1}, \ldots, x_{\ell}\right) ; d\right) \cong H^{*}\left(S(t) /\left(b_{1}, \ldots, b_{\ell}\right) ; d\right) .
$$

Therefore

$$
\begin{aligned}
H^{*}\left(G ; \mathrm{Sq}^{2}\right) & \cong H^{*}\left(P(y) \otimes \Lambda\left(x_{1}, \ldots, x_{\ell}\right) ; d\right) \\
& \cong H^{*}\left(P(y) \otimes S(t) /\left(b_{1}, \ldots, b_{\ell}\right) ; d\right) \cong H\left(\operatorname{gr} H^{*}(G / T ; \mathbb{Z} / 2) ; d\right) .
\end{aligned}
$$

Hence we have a spectral sequence

$$
H^{*}\left(G ; \mathrm{Sq}^{2}\right) \cong H\left(\operatorname{gr} H^{*}(G / T ; \mathbb{Z} / 2) ; d\right) \Rightarrow H\left(H^{*}(G / T ; \mathbb{Z} / 2) ; d\right) .
$$

Theorem 6.9. Let $k$ be an algebraically closed field in $\mathbb{C}$. For each compact connected simple Lie group $G$, Assumptions 4.1, 6.3, 6.7 are satisfied. If $G$ is simply connected, then there is an isomorphism

$$
W^{*}\left(G_{k}\right) \cong H^{*}\left(G / T ; \mathrm{Sq}^{2}\right) \cong \Lambda\left(z_{1}, \ldots, z_{s}\right), \quad \operatorname{deg}\left(z_{i}\right) \text { odd } .
$$


For $G=S O(n)$, we have $W^{*}\left(G_{k}\right) \cong H^{*}\left(G / T ; \mathrm{Sq}^{2}\right) \otimes \Lambda\left(x_{0}\right)$ with $\operatorname{deg}\left(x_{0}\right)=0$. Moreover $W^{*}\left(G_{k}\right)$ is isomorphic to

$$
\begin{cases}E(\mathrm{GP})_{4}^{*, *^{\prime}} \cong H\left(H^{*}\left(G ; \mathrm{Sq}^{2}\right) ; d_{3}\right) & \text { for } G=E_{6}, E_{7}, E_{8}, \\ E(\mathrm{GP})_{3}^{*, *^{\prime}} \cong H^{*}\left(G ; \mathrm{Sq}^{2}\right) & \text { otherwise. }\end{cases}
$$

Proof. We assume here Assumptions 6.3 and 6.7 which are shown for each simple Lie group $G$ in the next section.

Let $G$ be simply connected. (The case of $S O(n)$ is shown in $\S 7$ below; see e.g. Corollary 7.2.) From Assumption 6.7, the Gille-Pardon and the Balmer-Walter spectral sequences collapse because, from Lemma 6.6 , the $d_{r}$-image of $z_{i}$ must be some $z_{j}$ (modulo decomposable elements). However $\operatorname{deg}\left(z_{i}\right)-\operatorname{deg}\left(z_{j}\right)$ is even but $\operatorname{deg}\left(d_{r}\right)$ is odd, and this means $d_{r}=0$. Hence

$$
\operatorname{gr} W^{*}\left(G_{k}\right) \cong E(\mathrm{GP})_{4}^{*, *^{\prime}} \cong H\left(H^{*}\left(G ; \mathrm{Sq}^{2}\right) ; d_{3}\right) \cong \Lambda\left(z_{1}, \ldots, z_{s}\right)
$$

with $\operatorname{deg}\left(z_{i}\right)$ odd. Since $W^{*}\left(G_{k}\right)$ is a Hopf algebra (it is a product of truncated polynomial algebras), we obtain

$$
W^{*}\left(G_{k}\right) \cong \Lambda\left(z_{1}, \ldots, z_{s}\right) \cong \operatorname{gr} W^{*}\left(G_{k}\right) .
$$

Recall that $W^{*}\left(G_{k}\right) \cong W^{*}\left(G_{k} / T_{k}\right)$ is a subquotient of $H^{*}\left(G / T ; \mathrm{Sq}^{2}\right)$ from Corollary 4.2 (and is isomorphic to it if and only if Assumption 4.1 is satisfied).

On the other hand, by Lemma 6.8 , we know $H^{*}\left(G / T ; \mathrm{Sq}^{2}\right)$ is a subquotient of $H^{*}\left(G ; \mathrm{Sq}^{2}\right)$. Here we also assume that

(*) $\quad H^{*}\left(G / T ; \mathrm{Sq}^{2}\right)$ is a subquotient of $E(\mathrm{GP})_{4}^{*, *^{\prime}} \cong H\left(H^{*}\left(G ; \mathrm{Sq}^{2}\right) ; d_{3}\right)$.

(Of course $(*)$ holds when $d_{3}=0$. In the cases $G=E_{6}, E_{7}, E_{8},(*)$ is proved in the next section.) Then we get

$$
W^{*}\left(G_{k} / T_{k}\right) \cong W^{*}\left(G_{k}\right) \cong E(\mathrm{GP})_{4}^{*, *^{\prime}} \cong H^{*}\left(G / T ; \mathrm{Sq}^{2}\right)
$$

and Assumption 4.1 is satisfied.

Remark. Since $W^{*}\left(G_{k}\right)$ and $H^{*}\left(G / T ; \mathrm{Sq}^{2}\right)$ satisfy the Künneth formula from Corollary 4.3 , the above theorem holds for direct products of simple Lie groups (e.g. simply connected Lie groups). The explicit value of $\operatorname{deg}\left(z_{i}\right)$ is given in $\S 7$ below.

The homology $H^{*}\left(G ; \mathrm{Sq}^{2}\right)$ is computed more easily than $H^{*}\left(G / T\right.$; $\left.\mathrm{Sq}^{2}\right)$. However, in this paper, we use the ring structure of $H^{*}(G / T ; \mathbb{Z} / 2$ ) (for each simple Lie group) to deduce Lemma 3.1 (for Corollary 3.2 and Theorem 3.4) and to deduce Assumption 6.3. We hope to obtain alternative proofs that do not rely on the detailed ring structure of $H^{*}(G / T ; \mathbb{Z} / 2)$. 
Here we consider the map of spectral sequences induced from $G_{k} \rightarrow G_{k} / T_{k}$. Since $G_{k} / B_{k}$ is cellular, we have

$$
H^{2 *, *^{\prime}}\left(G_{k} / B_{k} ; \mathbb{Z} / 2\right) \cong H^{2 *, *^{\prime}}\left(G_{k} / T_{k} ; \mathbb{Z} / 2\right) \cong H^{2 *, *}\left(G_{k} / T_{k} ; \mathbb{Z} / 2\right) .
$$

We show $E(\mathrm{GP})_{r}^{*, *^{\prime}}=0$ for $* \neq *^{\prime}$. For dimensional reasons, we have isomorphisms

$$
E(\mathrm{GP})_{\infty}^{*, *^{\prime}}(G / T) \cong E(\mathrm{GP})_{3}^{*, *^{\prime}}(G / T) \cong E(\mathrm{GP})_{3}^{*, *}(G / T) \cong H^{*}\left(G / T ; \mathrm{Sq}^{2}\right) .
$$

From the above theorem, we see that the Balmer-Walter spectral sequence for $G_{k} / T_{k}$ also collapses

$$
E(\mathrm{GP})_{\infty}^{*, *^{\prime}}(G / T) \cong \operatorname{gr} E(\mathrm{BW})_{\infty}^{*, 4 c}(G / T) \cong \operatorname{gr} W^{*}\left(G_{k} / T_{k}\right) .
$$

Therefore the projection $G_{k} \rightarrow G_{k} / T_{k}$ induces isomorphisms $E(\mathrm{BW})_{r}^{*, *^{\prime}}(G / T)$ $\cong E(\mathrm{BW})_{r}^{*, *^{\prime}}(G)$ (for all $r>0$ ) of the Balmer-Walter spectral sequences, but not of the Gille-Pardon spectral sequences. In fact, for $* \neq *^{\prime}$ we see $E(\mathrm{GP})_{\infty}^{*, *^{\prime}}(G / T)=0$ but $E(\mathrm{GP})_{\infty}^{*, *^{\prime}}(G) \neq 0$ for some $* \neq *^{\prime}$ (see $\S 7$ below).

\section{$\S 7$. Simple Lie groups}

\section{$\S 7.1$. Classical Lie groups}

For the classical Lie groups, we will see Assumptions 6.3 and 6.7 hold. First, we consider the case $G=U(2 m+1)$. Its cohomology is

$$
\begin{aligned}
H^{*}(G ; \mathbb{Z} / 2) & \cong \Lambda\left(x_{1}, x_{3}, \ldots, x_{4 m+1}\right) \\
& \cong \Lambda\left(x_{1}\right) \otimes \bigotimes_{s=1}^{m} \Lambda\left(x_{4 s-1}, x_{4 s+1}\right) \quad \text { with } \quad \operatorname{Sq}^{2}\left(x_{4 s-1}\right)=x_{4 s+1}
\end{aligned}
$$

(Throughout this section, subscripts indicate degree, e.g, $\left|x_{i}\right|=i$ for convenience.)

Therefore we have the $\mathrm{Sq}^{2}$ homology

$$
H^{*}\left(G ; \mathrm{Sq}^{2}\right) \cong \Lambda\left(x_{1}\right) \otimes \bigotimes_{s=1}^{m} \Lambda\left(x_{4 s-1} x_{4 s+1}\right),
$$

which is (changing the degree so that $\left.\operatorname{deg}\left(x_{i}\right)=\frac{1}{2}(i-1)\right)$ isomorphic to

$$
\Lambda\left(u_{1}\right) \otimes H^{*}\left(G / T ; \mathrm{Sq}^{2}\right) \cong \Lambda\left(z_{0}\right) \otimes \otimes \Lambda\left(z_{4 s-1}\right), \quad \operatorname{deg}\left(z_{i}\right)=i .
$$

This result is also given by Kishimoto, Kono and Ohsita [Ki-Ko-Oh]. When $G=$ $U(2 m+2), H^{*}\left(G ; \mathrm{Sq}^{2}\right) \cong H^{*}\left(U(2 m+1) ; \mathrm{Sq}^{2}\right) \otimes \Lambda\left(x_{2 m+1}^{\prime}\right)$. The element $x_{2 m+1}^{\prime}$ corresponds to $z$ in the notation of [Ki-Ko-Oh]. For $G_{\mathrm{sc}}=S U(n)$, we get

$$
H^{*}\left(S U(n) ; \mathrm{Sq}^{2}\right) \cong H^{*}\left(U(n) ; \mathrm{Sq}^{2}\right) /\left(z_{0}\right)
$$

and the assumptions also hold. (See Corollary 2.4.) 
The case $G=\operatorname{Sp}(n)$ is easy. In fact, $H^{*}(G ; \mathbb{Z} / 2) \cong \Lambda\left(x_{3}, x_{7}, \ldots, x_{4 n-1}\right)$ and so $\mathrm{Sq}^{2}=0$. Hence $H^{*}\left(G ; \mathrm{Sq}^{2}\right) \cong \Lambda\left(x_{1}^{\prime}, \ldots, x_{2 n-1}^{\prime}\right)$ where $x_{2 i+1}^{\prime}$ corresponds to $x_{4 i+3}$ in $H^{*}(G ; \mathbb{Z} / 2)$.

Next we consider the case $G=S O(4 m+1)$. Then the mod 2 cohomology is written as (see for example $[\mathrm{Ni}]$ )

$$
\operatorname{gr} H^{*}(S O(4 m+1) ; \mathbb{Z} / 2) \cong \Lambda\left(x_{1}, x_{2}, \ldots, x_{4 m+1}\right)
$$

where $x_{s}^{2}=x_{2 s}$. We write $y_{2 \text { (odd) }}=x_{\text {odd }}^{2}$. Let us write

$$
\operatorname{gr} H^{*}(G ; \mathbb{Z} / 2) \cong \Lambda\left(y_{2}, y_{4}, \ldots, y_{4 m}\right) \otimes \Lambda\left(x_{1}, x_{3}, \ldots, x_{4 m+1}\right) .
$$

Here $\operatorname{gr} P(y) \cong \Lambda\left(y_{2}, y_{4}, \ldots, y_{4 m}\right)$ and $\operatorname{Sq}^{2}\left(y_{4 s+2}\right)=y_{4 s+4}$. Hence

$$
H^{*}\left(P(y) ; \mathrm{Sq}^{2}\right) \cong \bigotimes_{s=1}^{m} \Lambda\left(y_{4 s-2} y_{4 s}\right) .
$$

This result is also given by Kishimoto-Kono-Ohsita:

$$
H^{*}\left(S O(4 m+1) / T ; \mathrm{Sq}^{2}\right) \cong \bigotimes_{s=1}^{m} \Lambda\left(z_{4 s-1}, w_{4 s-1}\right)
$$

where $z_{4 s-1}$ (resp. $w_{4 s-1}$ ) corresponds to $x_{4 s-1} x_{4 s+1}$ (resp. $y_{4 s-2} y_{4 s}$ ). The cases of other $n$ are similar (see also [Ki-Ko-Oh]).

Now let $G=\operatorname{Spin}(n)$. The cohomology is given by

$$
\begin{aligned}
H^{*}(\operatorname{Spin}(4 m+1) ; \mathbb{Z} / 2) & \cong H^{*}(S O(4 m+1) ; \mathbb{Z} / 2) /\left(x_{1}, y_{1}\right) \otimes \Lambda(a) \\
& \cong \Lambda\left(y_{2}, y_{4}, \ldots, y_{4 m}\right) /\left(y_{2^{s}} \mid s \geq 1\right) \otimes \Lambda\left(x_{3}, \ldots, x_{4 m+1}\right) \otimes \Lambda(a)
\end{aligned}
$$

where $|a|=2^{t}-1$ for $2^{t-1}<4 m \leq 2^{t}$ ([Mi-Ni], [Tod]). Then we can prove that $H^{*}\left(G ; \mathrm{Sq}^{2}\right)$ is isomorphic to

$$
H^{*}\left(S O(n) ; \mathrm{Sq}^{2}\right) /\left(z_{0}, w_{2^{s}-1} \mid s \geq 1\right) \otimes\left(\bigotimes_{s=1}^{t-2} \Lambda\left(y_{2^{s}-1}^{\prime}\right)\right) \otimes \Lambda\left(a_{2^{t-1}-1}^{\prime}\right)
$$

where $w_{2^{s-1}}$ (resp. $\left.y_{2^{s-1}-1}^{\prime}, a_{2^{t-1}-1}^{\prime}\right)$ corresponds to $y_{2^{s}-2} y_{2^{s}}$ (resp. $\left.y_{2^{s}-2}, a\right)$. Identifying $y_{2^{s}-1}^{\prime}=w_{2^{s-1}}$ and $a_{2^{t-1}-1}^{\prime}=w_{2^{i-1}-1}$, we have an isomorphism

$$
H^{*}\left(S O(n) ; \mathrm{Sq}^{2}\right) \cong H^{*}\left(\operatorname{Spin}(n) ; \mathrm{Sq}^{2}\right) \otimes \Lambda\left(z_{0}\right) \text {. }
$$

Proposition 7.1. Let $G$ be a simply connected simple classical group. Then Assumptions 6.3 and 6.7 are satisfied.

Proof. Assumption 6.7 is satisfied from the above arguments if Assumption 6.3 is satisfied. We will prove Assumption 6.3 for $G=\operatorname{Spin}(4 m+1)$. The other cases follow from the naturality of maps, e.g. $S U(n) \rightarrow \operatorname{Spin}(2 n)$. 
Let $G^{\prime}=\operatorname{Spin}(4 m-3)$ and suppose the assumption holds for $G^{\prime}$. Assume $4 m \neq 2^{s}$. Then

$$
\begin{gathered}
H^{*}(G ; \mathbb{Z} / 2) \cong H^{*}\left(G^{\prime} ; \mathbb{Z} / 2\right) \otimes \Lambda\left(y_{4 m-2}, y_{4 m}, x_{4 m-1}, x_{4 m+1}\right) \quad \text { with } \\
\operatorname{Sq}^{2}\left(y_{4 m-2}\right)=y_{4 m}, \quad \operatorname{Sq}^{2}\left(x_{4 m-1}\right)=x_{4 m+1} .
\end{gathered}
$$

By Totaro's theorem, $d_{2}\left(y_{4 m-2}\right)=y_{4 m}$. In the Gille-Pardon spectral sequence, if $d_{2}\left(x_{4 m-1}\right) \neq x_{4 m+1}$, then $d_{2}\left(x_{4 m-1}\right)=0$ by Lemma 6.6. We have f.deg $\left(x_{4 m-1}\right)=2 m-1$ but the first degree of a differential is always odd. Hence $x_{4 m-1}$ is a permanent cycle. However this element does not exist in $H^{*}\left(G / T ; \mathrm{Sq}^{2}\right) \cong$ $H^{*}\left(G ; \mathrm{Sq}^{2}\right)$. Since $W^{*}\left(G_{k}\right)$ is a subquotient of $H^{*}\left(G / T ; \mathrm{Sq}^{2}\right)$, this is a contradiction. So $d_{2}\left(x_{4 m-1}\right)=x_{4 m+1}$.

When $4 m=2^{s}$, the different parts (from the case of $4 m \neq 2^{s}$ ) are even-dimensional ring generators. By Totaro's theorem, also $d_{2}\left(y_{i}\right)=\operatorname{Sq}^{2}\left(y_{i}\right)$. Thus

$$
E(\mathrm{GP})_{3}^{*, *^{\prime}} \cong H^{*}\left(G ; \mathrm{Sq}^{2}\right) \cong \Lambda\left(u_{1}, \ldots, u_{r}\right), \quad \operatorname{deg}\left(u_{i}\right) \text { odd. }
$$

The following fact is also shown in Corollary 2.4.

Corollary 7.2. We have an isomorphism

$$
W^{*}(S O(n)) \cong W^{*}(\operatorname{Spin}(n)) \otimes \Lambda\left(z_{0}\right) \text {. }
$$

Proof. By Proposition 7.1 and the naturality of the map $\operatorname{Spin}(n) \rightarrow S O(n)$,

$$
E(\mathrm{GP})_{3}^{*, *^{\prime}} \cong H^{*}\left(G ; \mathrm{Sq}^{2}\right) \cong \Lambda\left(z_{0}, u_{1}, \ldots, u_{r}\right) .
$$

We only need to see $d_{r}\left(z_{0}\right)=0$, which follows from the fact that f.deg $\left(d_{r}\left(z_{0}\right)\right)=$ $1 \bmod (4)$ but f.deg $\left(u_{i}\right)=-1 \bmod (4)$.

\section{§7.2. Exceptional Lie groups $G_{2}$ and $F_{4}$}

The cohomology of $G_{2}$ is

$$
\operatorname{gr} H^{*}\left(G_{2} ; \mathbb{Z} / 2\right) \cong \Lambda\left(y_{6}, x_{3}, x_{5}\right) \quad \text { with } \quad \operatorname{Sq}^{2}\left(x_{3}\right)=x_{5}
$$

We have the natural inclusion $S U(3) \subset G_{2}$. Recall $H^{*}(S U(3) ; \mathbb{Z} / 2) \cong \Lambda\left(x_{3}, x_{5}\right)$ and $d_{2}\left(x_{3}\right)=x_{5}$ in the Gille-Pardon spectral sequence. Hence in $H^{*}\left(G_{2} ; \mathbb{Z} / 2\right)$, we have $d_{2}\left(x_{3}\right)=x_{5}$, and

$$
H^{*}\left(G_{2} ; \mathrm{Sq}^{2}\right) \cong \Lambda\left(z_{3}, y_{3}^{\prime}\right)
$$

where $z_{3}$ (resp. $y_{3}^{\prime}$ ) corresponds to $x_{3} x_{5}$ (resp. $y_{6}$ ). The cohomology of $F_{4}$ is

$$
H^{*}\left(F_{4} ; \mathbb{Z} / 2\right) \cong H^{*}\left(G_{2} ; \mathbb{Z} / 2\right) \otimes \Lambda\left(x_{15}, x_{23}\right) .
$$


Hence $H^{*}\left(F_{4} ; \mathrm{Sq}^{2}\right) \cong H^{*}\left(G_{2} ; \mathrm{Sq}^{2}\right) \otimes \Lambda\left(x_{7}^{\prime}, x_{11}^{\prime}\right)$ where $x_{7}^{\prime}$ (resp. $\left.x_{11}^{\prime}\right)$ corresponds to $x_{15}$ (resp. $x_{23}$ ). (See also [Ki-Oh].) Thus Assumptions 6.3 and 6.7 are satisfied in these cases.

\section{§7.3. Exceptional Lie groups $E_{6}, E_{7}, E_{8}$}

First consider the case $G=E_{6}$. The cohomology of $E_{6}$ is

$$
\operatorname{gr} H^{*}\left(E_{6} ; \mathbb{Z} / 2\right) \cong \Lambda\left(y_{6}, x_{3}, x_{5}, x_{9}, x_{15}, x_{17}, x_{23}\right),
$$

where $\operatorname{Sq}^{2}\left(x_{i}\right)=x_{i+2}$. We easily see that

$$
H^{*}\left(G ; \mathrm{Sq}^{2}\right) \cong \Lambda\left(y_{3}^{\prime}, z_{3}, x_{4}^{\prime}, z_{15}, x_{11}^{\prime}\right)
$$

with the notation similar to the above cases of $G_{2}$ and $F_{4}$ (e.g. $x_{4}^{\prime}=x_{9}, z_{15}=$ $\left.x_{15} x_{17}, x_{11}^{\prime}=x_{23}\right)$.

The spectral sequence in Lemma 6.8 does not collapse for the following reason. In $H^{*}(G / T ; \mathbb{Z} / 2)$, we know $\mathrm{Sq}^{2}\left(y_{6}\right)=c_{4}([\mathrm{Ki}-\mathrm{Oh}, \S 4]$, [Ko-Is, Theorem 5.9], where $y_{6}$ is written as $\left.\gamma_{3}\right)$. So $d_{3}\left(y_{3}^{\prime}\right)=x_{4}^{\prime}$, and we have

$$
H^{*}\left(G / T ; \mathrm{Sq}^{2}\right) \cong \Lambda\left(z_{3}, v_{7}, z_{15}, x_{11}^{\prime}\right)
$$

where $v_{7}$ corresponds to $y_{6} x_{9}$. Indeed, this result is also given in [Ki-Oh].

Now we return to the Gille-Pardon spectral sequence

$$
E(\mathrm{GP})_{3}^{*, *^{\prime}} \cong \begin{cases}\Lambda\left(y_{3}^{\prime}, z_{3}, x_{4}^{\prime}, z_{15}, x_{11}^{\prime}\right) & \text { if } d_{2}\left(x_{15}\right)=y_{17} \\ \Lambda\left(y_{3}^{\prime}, z_{3}, x_{4}^{\prime}, x_{7}^{\prime}, x_{8}^{\prime}, x_{11}^{\prime}\right) & \text { otherwise }\end{cases}
$$

If $d_{3}\left(y_{6}\right) \neq x_{9}$ (i.e. $d_{3}\left(y_{3}^{\prime}\right) \neq x_{4}^{\prime}$ ), then $x_{9}=x_{4}^{\prime}$ is a permanent cycle in the Gille-Pardon and the Balmer-Walter spectral sequences for the following reason. In $E(\mathrm{GP})_{r}^{*, *^{\prime}}$ the degree of the differential is $\operatorname{deg}\left(d_{r}\right)=(1, r-1)$ and $w\left(d_{r}\right)=r-2$. We have $w\left(z_{3}\right)=w\left(x_{3} x_{5}\right)=2$ but $w\left(x_{4}^{\prime}\right)=w\left(x_{9}\right)=1$, and hence there is no differential such that $d\left(z_{3}\right)=x_{4}^{\prime}$ in the Gille-Pardon spectral sequence. In the Balmer-Walter spectral sequence, if $d_{r}\left(x_{4}\right)=u_{i} \neq 0$, then from Lemma 6.6, we see that $u_{i}=x_{i}^{\prime}$ or $z_{i}$. Moreover $\operatorname{deg}\left(u_{i}\right)-\operatorname{deg}\left(x_{4}^{\prime}\right) \neq 1 \bmod (4)$ from the degree of $d_{r}$ and this is impossible. Hence $x_{4}^{\prime}$ is a permanent cycle.

However $W^{*}(G)$ is a subquotient of $H^{*}\left(G / T ; \mathrm{Sq}^{2}\right)$, where there is no generator with $\operatorname{dim}=4$. This is a contradiction and we have proved that $d_{3}\left(y_{6}\right)=x_{9}$ (i.e. $\left.d_{3}\left(y_{3}^{\prime}\right)=x_{4}^{\prime}\right)$.

We can prove $d_{2}\left(x_{15}\right)=x_{17}$ similarly. Therefore we have Assumptions 6.3 and 6.7 for $G=E_{6}$ :

$$
E(\mathrm{GP})_{4}^{*, *^{\prime}} \cong \Lambda\left(z_{3}, v_{7}, z_{15}, x_{11}^{\prime}\right) .
$$

(In fact we have showed $(*)$ in the proof of Theorem 6.9.) 
Next we consider the case $G=E_{7}$. The cohomology of $E_{7}$ is

$$
\operatorname{gr} H^{*}\left(E_{7} ; \mathbb{Z} / 2\right) \cong \operatorname{gr} H^{*}\left(E_{6} ; \mathbb{Z} / 2\right) \otimes \Lambda\left(y_{10}, y_{18}, x_{27}\right) .
$$

Hence we easily see that

$$
\begin{aligned}
H^{*}\left(G / T ; \mathrm{Sq}^{2}\right) & \cong H^{*}\left(E_{6} / T ; \mathrm{Sq}^{2}\right) \otimes \Lambda\left(y_{5}^{\prime}, y_{9}^{\prime}, x_{13}^{\prime}\right) \\
& \cong \Lambda\left(v_{7}, y_{5}^{\prime}, y_{9}^{\prime}, z_{3}, z_{15}, x_{11}^{\prime}, x_{13}^{\prime}\right)
\end{aligned}
$$

where $y_{5}^{\prime}=y_{10}, y_{9}^{\prime}=y_{18}, x_{13}^{\prime}=x_{27}$.

Finally, we consider the case $G=E_{8}$. The cohomology of $E_{8}$ is

$$
\begin{aligned}
& \operatorname{gr} H^{*}\left(E_{8} ; \mathbb{Z} / 2\right) \cong \operatorname{gr} H^{*}\left(E_{7} ; \mathbb{Z} / 2\right) \otimes \Lambda\left(y_{12}, y_{24}, y_{20}, y_{30}, x_{29}\right) \\
& \cong \Lambda\left(y_{i} \mid i=6,12,24,10,20,18,30\right) \otimes \Lambda\left(x_{j} \mid j=3,5,9,15,17,23,27,29\right) .
\end{aligned}
$$

The Steenrod operation acts as $\mathrm{Sq}^{2}\left(y_{i}\right)=y_{i+2}, \mathrm{Sq}^{2}\left(x_{i}\right)=x_{i+2}$ and $\mathrm{Sq}^{2}\left(y_{12} y_{10}\right)$ $=y_{24}$ (in fact, $y_{24}=y_{12}^{2}$ ). Hence we can compute

$$
H^{*}\left(G / T ; \mathrm{Sq}^{2}\right) \cong \Lambda\left(v_{7}, w_{23}, w_{19}, y_{15}^{\prime}, z_{3}, z_{15}, x_{11}^{\prime}, z_{27}\right)
$$

where $w_{23}=y_{10} y_{12} y_{24}, y_{15}^{\prime}=y_{30}, z_{27}=x_{27} x_{29}$. Ohsita first computed this homology by using the $\mathrm{Sq}^{2}$-algebra structure of $H^{*}(G / T ; \mathbb{Z} / 2)$, and of course, our result coincides with his. We can easily show $d_{2}\left(x_{27}\right)=x_{29}$ in the Gille-Pardon spectral sequence and we obtain Assumptions 6.3 and 6.7.

\section{Acknowledgements}

I am grateful to Masaharu Kaneda, Michishige Tezuka, Burt Totaro and Marcus Zibrowius for their useful suggestions and comments. I also thank the referees for the many valuable suggestions of improvement, in particular, for the contents of $\S 2$ in this paper.

\section{References}

[Ar1] S. Araki, Differential Hopf algebras and the cohomology mod 3 of the compact exceptional groups $E_{7}$ and $E_{8}$, Ann. of Math. 73 (1961), 404-436. Zbl 0147.23303 MR 0124052

[Ar2] Generalized cohomology theories, Kinokuniya, Tokyo, 1975, xvi+317 pp. (in Japanese).

[Ars] J. Arason, Der Wittring projektiver Räume, Math. Ann. 253 (1980), 205-212. Zbl 0431.10011 MR 0597829

[At] M. Atiyah, $K$-theory and reality, Quart. J. Oxford 17 (1966), 367-386.

[Ba] P. Balmer, Witt groups, in Handbook of K-theory, Springer, Berlin, 2005, 539-576. Zbl 1115.19004 MR 2181829 
[Ba-Ca1] P. Balmer and B. Calmès, Geometric description of the connecting homomorphism for Witt groups, Doc. Math. 14 (2009), 525-550. Zbl 1197.19003 MR 2565903

[Ba-Ca2] _ _ Witt groups of Grassmann varieties, J. Algebraic Geom. 21 (2012), 601-642. Zbl 1273.14098 MR 2957690

[Ba-Wa] P. Balmer and C. Walter, A Gersten-Witt spectral sequence for regular schemes, Ann. Sci. École Norm. Sup. 35 (2002), 127-152. Z Zbl 1012.19003 MR 1886006

[Bl-Og] S. Bloch and A. Ogus, Gersten's conjecture and the homology of schemes, Ann. Sci. École Norm. Sup. 7 (1974), 181-202. Z Zl 0307.14008 MR 0412191

[Bo] A. Borel, Linear algebraic groups, 2nd ed., Grad. Texts in Math. 126, Springer, New York, 1991. Zbl 0726.20030 MR 1102012

[Br] P. Brosnan, Steenrod operations in Chow theory, Trans. Amer. Math. Soc. 355 (2003), 1869-1903. Zbl 1045.55005 MR 1953530

[Ca-Fa] B. Calmès and J. Fasel, Trivial Witt groups of flag varieties, J. Pure Appl. Algebra 216 (2012), 404-406. Zbl 1235.19002 MR 2835204

[Ca-Ho] B. Calmès and J. Hornbostel, Witt motives, transfers and dévissage, http://www math.uiuc.edu/K-theory/0786/ (2006).

[Co-Ho-Ka] J.-L. Colliot-Thélène, R. Hoobler and B. Kahn, The Bloch-Ogus-Gabber theorem, in Algebraic K-theory (Toronto, 1996), Field Inst. Comm. 16, Amer. Math. Soc., 1997, 31-94. Zbl 0911.14004 MR 1466971

[Fu] M. Fujii, KO-group of projective spaces, Osaka J. Math. 4 (1967), 141-149. Zbl 0153.25201 MR 0219060

[Gi] S. Gille, A graded Gersten-Witt complex for schemes with a dualizing complex and the Chow group, J. Pure Appl. Algebra 208 (2007), 391-419. Zbl 1127.19005 MR 2277683

[Ha] S. Hara, Note on $K O$-theory of $B O(n)$ and $B U(n)$, J. Math. Kyoto Univ. 31 (1991), 487-493. Zbl 0735.19006 MR 1121175

[Ho] J. Hornbostel, $\mathbb{A}^{1}$-representability of hermitian $K$-theory and Witt groups, Topology 44 (2005), 661-687. Zbl 1078.19004 MR 2122220

[Ka] R. Kane, The homology of Hopf spaces, North-Holland Math. Library 40, NorthHolland, Amsterdam, 1988. Zbl 0651.55001 MR 0961257

[Ki-Ko-Oh] D. Kishimoto, A. Kono and A. Ohsita, KO-theory of flag manifolds, J. Math. Kyoto Univ. 44 (2004), 217-227. Zbl 1065.55001 MR 2062716

[Ki-Oh] D. Kishimoto and A. Ohsita, KO-theory of exceptional flag manifolds, J. Math. Kyoto Univ. 53 (2013), 673-692. Zbl 06212905 MR 3102565

[Ko-Ha] A. Kono and S. Hara, KO-theory of complex Grassmannians, J. Math. Kyoto Univ. 31 (1991), 827-833. Zbl 0748.57015 MR 1127102

[Ko-Is] A. Kono and K. Ishitoya, Squaring operations in mod 2 cohomology of quotients of compact Lie groups by maximal tori, in Algebraic topology (Barcelona, 1986), Lecture Notes in Math. 1298, Springer, 1987, 192-206. Zbl 0656.57025 MR 0928833

[Mi-Ni] M. Mimura and T. Nishimoto, Hopf algebra structure of Morava $K$-theory of exceptional Lie groups, in Recent progress in homotopy theory (Baltimore, MD, 2000), Contemp. Math. 293, Amer. Math. Soc., 2002, 195-231. Zbl 1011.57011 MR 1890737

[Ne] A. Nenashev, Gysin maps in Balmer-Witt theory, J. Pure Appl. Algebra 211 (2007), 203-221. Zbl 1140.11024 MR 2333767

[Ni] T. Nishimoto, Higher torsion in Morava K-theory of $S O(m)$ and $\operatorname{Spin}(m)$, J. Math. Soc. Japan 52 (2001), 383-394. Zbl 0983.55004 MR 1815140

[Oh] A. Ohsita, On KO-theory of flag manifolds of exceptional Lie groups, unpublished (2008). 
[Or-Vi-Vo] D. Orlov, A. Vishik and V. Voevodsky, An exact sequence for $K_{*}^{M} / 2$ with applications to quadratic forms, Ann. of Math. 165 (2007), 1-13. Zbl 1124.14017 MR 2276765

[Pa-Wa] I. Panin and C. Walter. On the motivic commutative ring spectrum BO, www.math. uiuc.edu/K-theory/0978 (2010).

[Pa] W. Pardon. The filtered Gersten-Witt resolution for regular schemes, www.math. uiuc.edu/K-theory/0419 (2000).

[Sch] M. Schlichting, The Mayer-Vietoris principle for Grothendieck-Witt groups of schemes, Invent. Math. 179 (2010), 349-433. Zbl 1193.19005 MR 2570120

[Sch-Tr] M. Schlichting and G. Tripathi, Geometric representation of Hermitian K-theory, preprint (2012).

[Tod] H. Toda, On the cohomology ring of some homogeneous spaces, J. Math. Kyoto Univ. 15 (1975), 185-199. Zbl 0323.57025 MR 0380856

[To] B. Totaro, Non-injectivity of the map from the Witt group of a variety to the Witt group of its function field, J. Inst. Math. Jussieu 2 (2003), 483-493. Zbl 1038.19004 MR 1990223

[Vo1] V. Voevodsky, The Milnor conjecture, http://www.math.uiuc.edu/K-theory/0170/ (1996).

[Vo2] _ Motivic cohomology are isomorphic to higher Chow groups, http://www. math.uiuc.edu/K-theory/378/ (1999).

[Vo3] $\quad$ L Reduced power operations in motivic cohomology, Publ. Math. IHES 98 (2003), 1-57. Zbl 1057.14027 MR 2031198

[Ya1] N. Yagita, Motivic cohomology of quadrics and coniveau spectral sequence, J. K-theory 6 (2010), 547-589. Zbl 1218.14015 MR 2746285

[Ya2] Note on the mod $p$ motivic cohomology of algebraic groups, Kyushu J. Math. 66 (2012), 245-259. Zbl 06136000 MR 3051335

[Ya3] A note on the Witt group and the KO-theory of complex Grassmannians, J. K-theory 9 (2012), 161-175. Zbl 1250.19006 MR 2887204

[Zi] M. Zibrowius, Witt groups of complex cellular varieties, Doc. Math. 16 (2011), 465511. Zbl 05947061 MR 2823367 